\title{
Simulating Evolution in Asexual Populations with Epistasis
}

\section{Ramon Diaz-Uriarte}

\begin{abstract}
I show how to use OncoSimulR, software for forward-time genetic simulations, to simulate evolution of asexual populations in the presence of epistatic interactions. This chapter emphasizes the specification of fitness and epistasis, both directly (i.e., specifying the effects of individual mutations and their epistatic interactions) and indirectly (using models for random fitness landscapes).
\end{abstract}

Key words Simulation, Epistasis, Fitness landscape, Evolution, Mutation, Fitness

\section{Introduction}

Here we illustrate the use of the Bioconductor package OncoSimulR for simulating evolution of asexual populations with epistasis. OncoSimulR [11] implements forward-time genetic simulations in asexual populations, using biallelic loci. Fitness can be defined either directly (by specifying the fitness landscape, or the map between genotypes and fitness), as shown in Subheadings 2.2.1 and 2.2.2, or by specifying epistatic interactions directly as shown in Subheadings 2.2.4-2.2.7. Simulations use a continuous time model, and employ the state-of-the-art BNB algorithm of Mather et al. [23]. Some previous uses of OncoSimulR include the study of the sensitivity of cancer progression models to reciprocal sign epistasis [12], the predictability of cancer evolution [13, 19], and somatic mutation in plants [33].

\section{Methods}

Using OncoSimulR for the simulation of evolutionary processes involves:

1. Installing (if needed) and loading OncoSimulR. 
2.1 Installing and Loading OncoSimulR
2. Choosing the mapping between genotypes and fitness. It is at this stage that we specify epistasis.

3. Choosing the details of the evolutionary model, including growth models and the specifics of the mutation process.

4. Running simulations until pre-specified conditions are reached.

The second and third steps can be decided in any order, but they come necessarily (logically and chronologically) before the last. Since the focus of this book is on epistasis it is thus preferable to order the above steps as follows:

1. Installing (if needed) and loading OncoSimulR (Subheading 2.1).

2. Specifying epistasis, which can be done either:

- specifying epistasis indirectly, which includes possibly using models for random fitness landscapes (Subheadings 2.2.1 and 2.2.2) or

- $\quad$ specifying epistasis directly (Subheadings 2.2.4-2.2.7).

3. Simulating evolution, which involves:

- Choosing growth models (Subheading 2.3.1).

- Defining mutation rates and possible mutator genes (Subheading 2.3.2).

- Running simulations until pre-specified conditions are met (Subheadings 2.3.3-2.3.7).

The next sections are structured following the above order. The code shown below illustrates the usage of the most important functionality, and we discuss the key options for specifying fitness and epistasis; not all options used are discussed, though (see the package vignette and function documentation for details).

If OncoSimulR is not installed, we must install it. Note that we are using the development version of OncoSimulR, from Bioconductor 3.11, that runs under what will become R-4.0. Please refer to the specific instructions for installing $\mathrm{R}$ for your operating system (https://cran.r-project.org). Once we have installed R-4.0 we can install packages for Bioconductor 3.11 (details about the release and development version of Bioconductor are available from https://www.bioconductor.org/developers/how-to/useDevel/).

We are now ready to install OncoSimulR following https:// www.bioconductor.org/packages/devel/bioc/html/ OncoSimulR.html: 


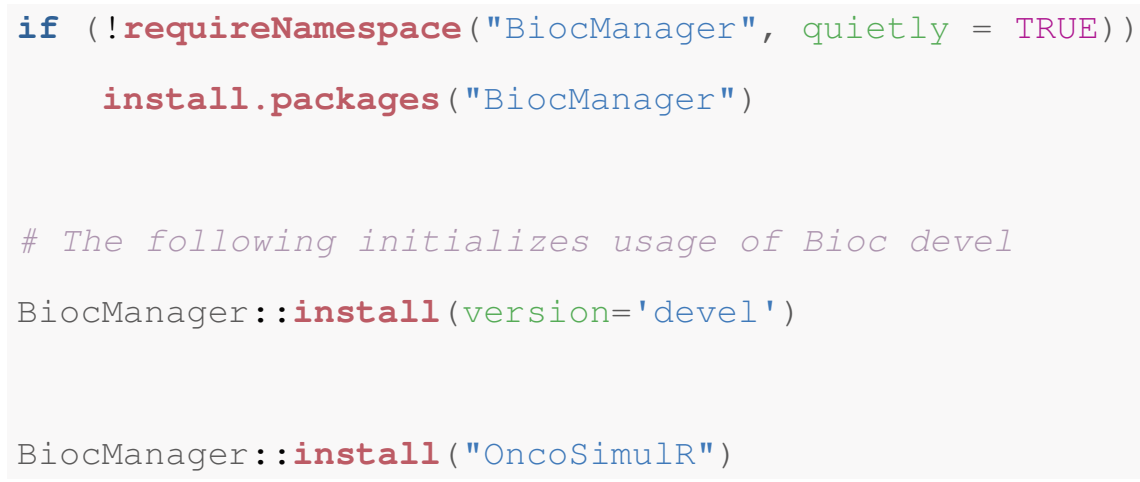

Installation of OncoSimulR only needs to be carried out once (more precisely, whenever the versions of OncoSimulR or of $\mathrm{R}$ change).

Once OncoSimulR is installed, in every $R$ session where we want to use it, we must load the package. Here we load it and also verify its version (at least 2.17.1)

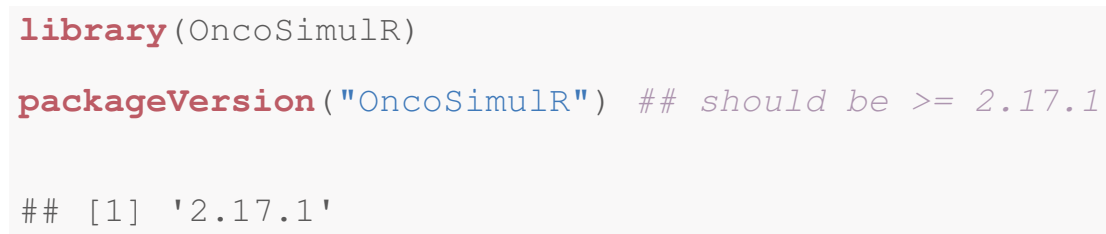

\subsection{Specifying Epistasis}

2.2.1 Specifying Epistasis Indirectly: Explicit Mapping from Genotypes to Fitness
We can either specify epistasis indirectly, as shown in Subheadings 2.2.1 and 2.2.2, or directly, by specifying the fitness effects of mutations on genes (Subheadings 2.2.3-2.2.7), thus having full control over the specification of epistatic interactions (see also Notes 1 and 2).

We can specify the mapping between genotypes and fitness by explicitly indicating what the fitness of all possible genotypes (or all genotypes with non-zero fitness) is. Here, epistasis is not specified directly, but indirectly. For instance, we could specify a four genotype model with sign epistasis as:

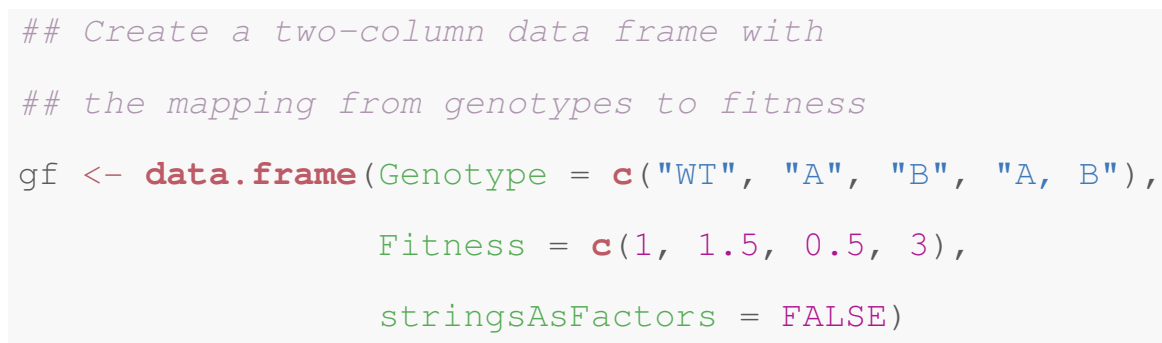




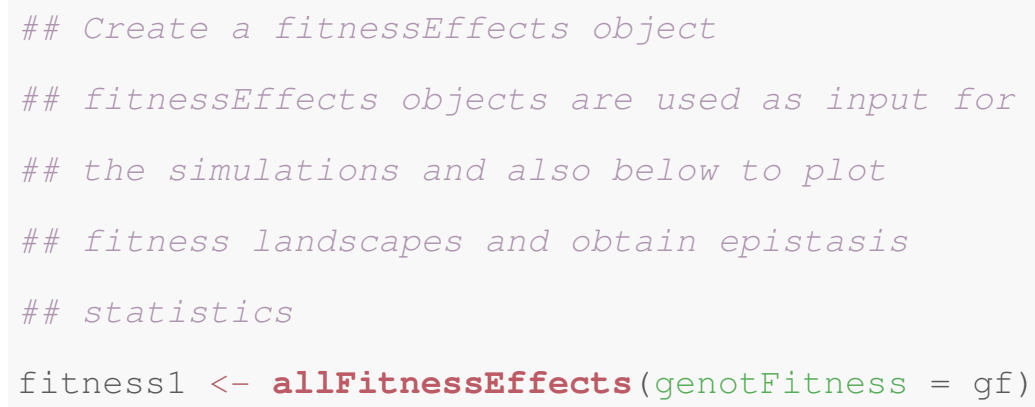

We can plot the fitness of the four genotypes by calling either plotFitnessLandscape or plot as follows (figure not shownbut see below, Subheading 2.2.2, for a fitness landscape plot of a House of Cards model):

plotFitnessLandscape (fitness 1 )

And we can compute some epistasis statistics using the function Magellan_stats that calls code provided by MAGELLAN [4] (more precisely, function f1_statistics). From the set of statistics provided by MAGELLAN, we only want the fraction of pairs of loci that have no epistasis, magnitude epistasis, sign epistasis, reciprocal sign epistasis, and $\gamma$, the correlation in fitness effects between genotypes that differ by one locus (averaged over the fitness landscape) (see details in [4] and [14]). Since we are only interested in some of the output provided by the Magellan_stats function, we write a simple wrapper to Magellan_stats (where use_log controls whether we take the log of the fitness values before computing the epistasis statistics):

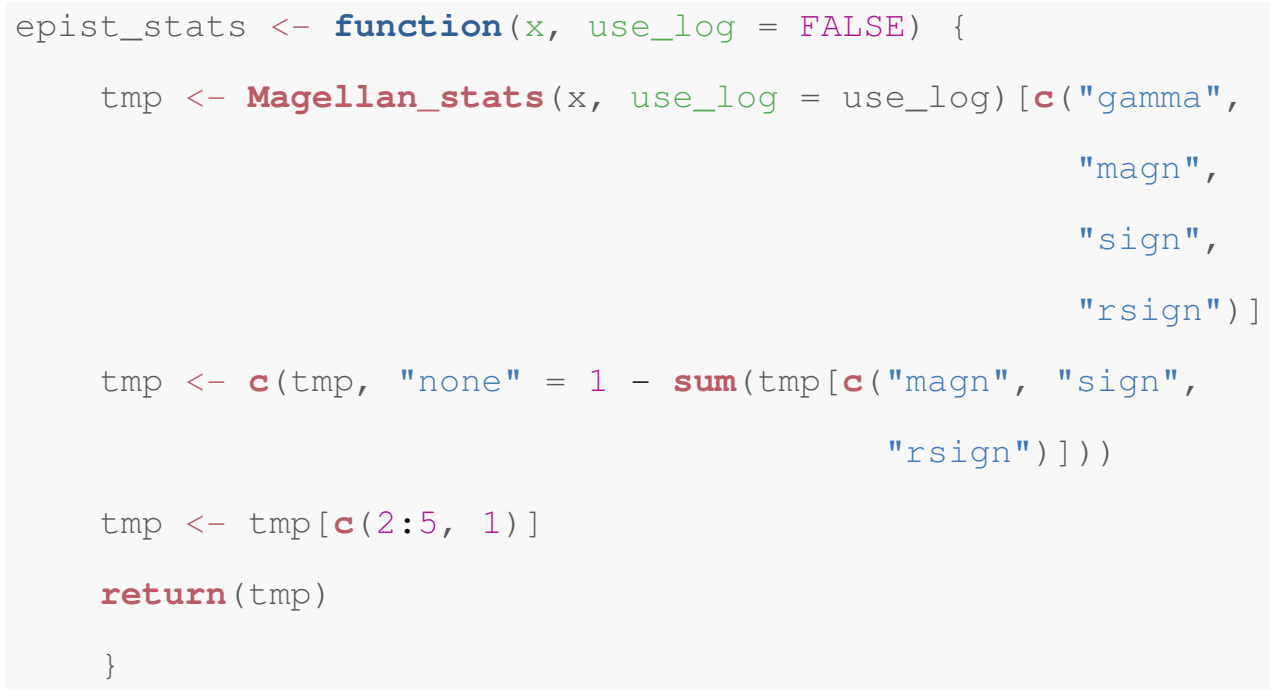


Now we can compute the epistasis statistics as

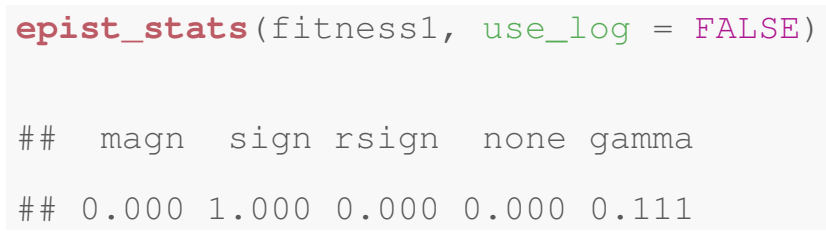

which shows that there is only sign epistasis in the model.

Computing the epistasis statistics on the log-transformed fitness data does not change the estimates of epistasis (it does change the estimate of $\gamma$, though), since sign epistasis is not affected by monotonic transformations:

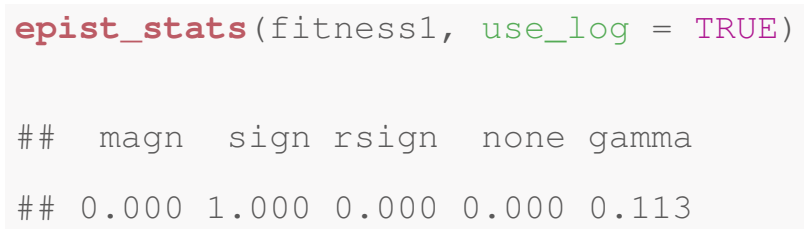

(Using use_log = TRUE is discussed with more detail below: see Subheading 2.2.3.)

\subsubsection{Specifying}

Epistasis Indirectly: Using

Models for Fitness

Landscapes
Alternatively, the mapping between genotypes and fitness can be done according to different random fitness landscapes models, which are characterized by different degrees of epistasis (see $[4,14,35])$. We will use function $r \mathrm{fitness}$ that allows us to use the Rough Mount Fuji (RMF) model [1, 14, 28, 35], which includes as limit cases both a fully additive model $[14,35]$ and the House of Cards model [14, 22, 35], and the NK (or LK) model $[14,20,21,35]$. It must be noted that the values returned by rfitness are to be interpreted as log-fitness values (and this is why, in the calls in this section, we call the function epist_stats without log-transforming the fitness values; see also Subheading 2.2.3 for further discussion of using use_log = TRUE when calling epist_stats).

In the examples that follow, and for ease of representation, we will only use four loci. We will first use a fully additive and deterministic model. The additive model is sometimes also called a multiplicative model, as it becomes additive in the log scale; the multiplicative effect on fitness that each mutation has does not depend on the state of other genes (see $[4,14])$.

We can generate deterministic, additive models as special cases of Rough Mount Fuji models without noise [28, 35]. In the example below, the genotype with all genes mutated has maximum fitness (reference $=\max$ ), and all genotypes have the same decrease in fitness per unit increase in Hamming distance from the genotype with maximum fitness $(\mathrm{c}=0.5)$ ( see also Note 3 ). 


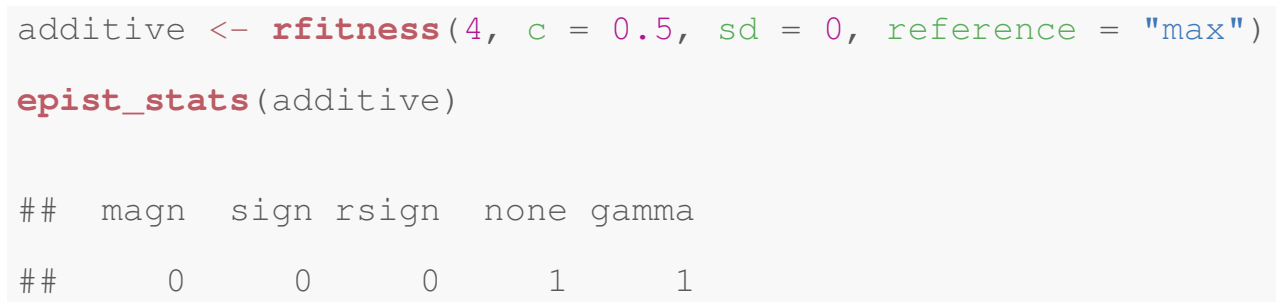

As above, we could plot the fitness landscape calling either plotfitnesslandscape or plot as (figure not shown-but see below for a fitness landscape plot of a House of Cards model):

The other extreme of the RMF model is the House of Cards model $[14,22,35]$ that leads to maximally rugged fitness landscapes: in the House of Cards model the (log) fitness of each genotype is obtained, independently for each genotype, from some underlying distribution (normal, in this case):

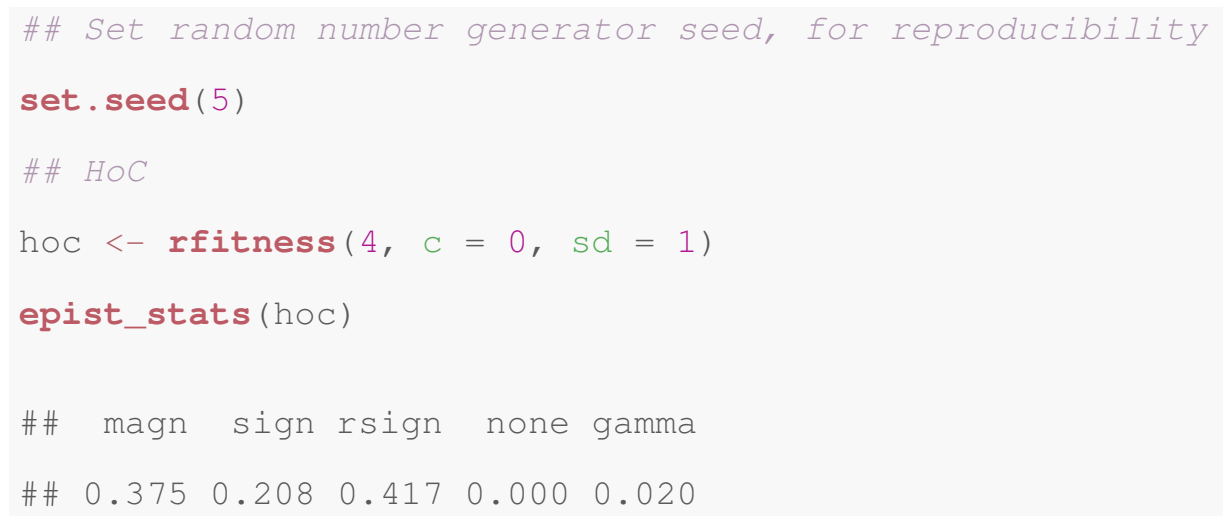

The plot of the fitness landscapes can be obtained as

and is shown in Fig. 1.

The RMF model [1, 14, 28, 35] is a combination of an additive model (the "Mount Fuji" part, with the peak on the genotype of maximal fitness) and a House of Cards model (the random component). Thus, it has intermediate behavior in terms of epistasis: 


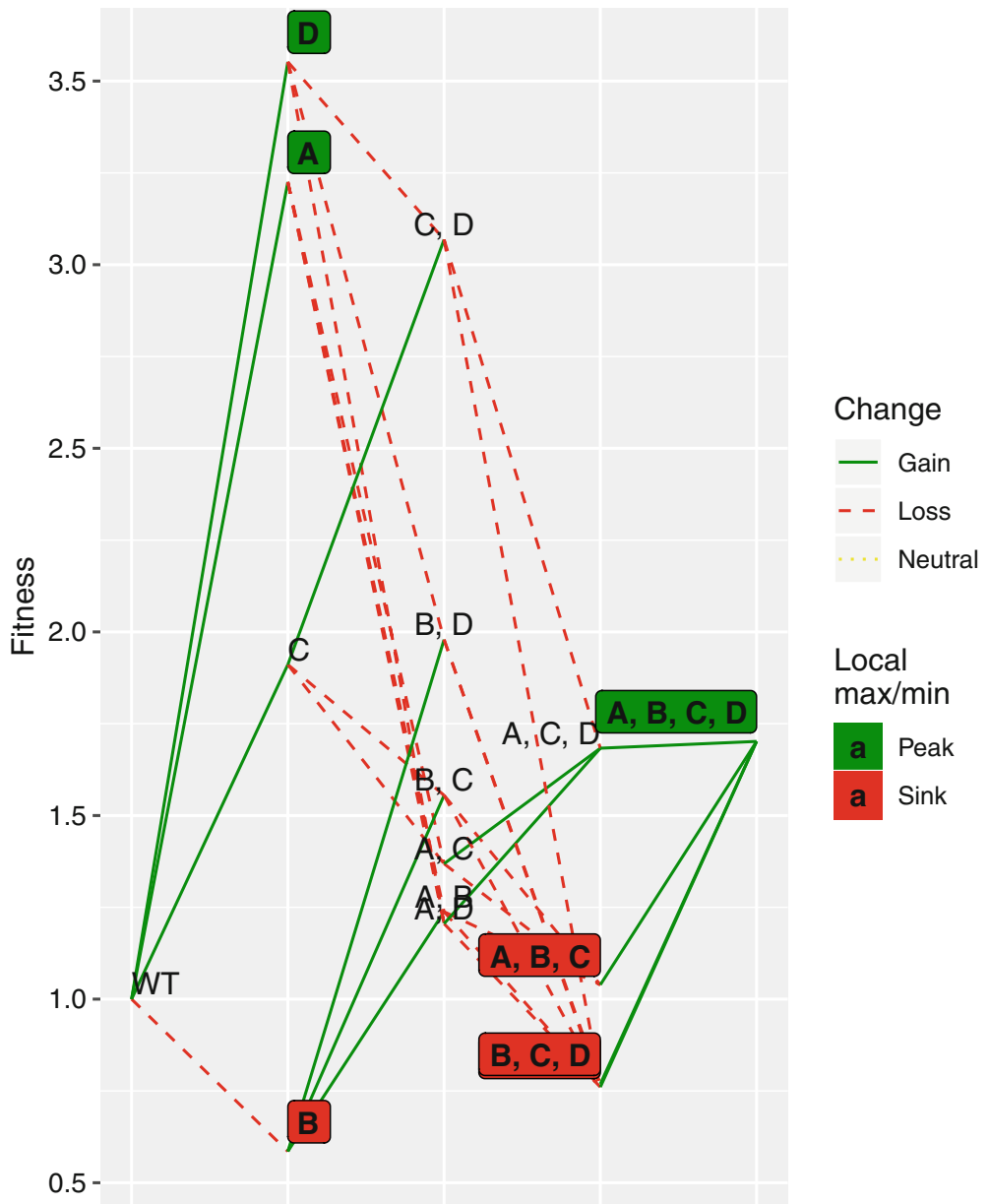

Fig. 1 Plot of the fitness landscape for the House of Cards model in Subheading 2.2.2

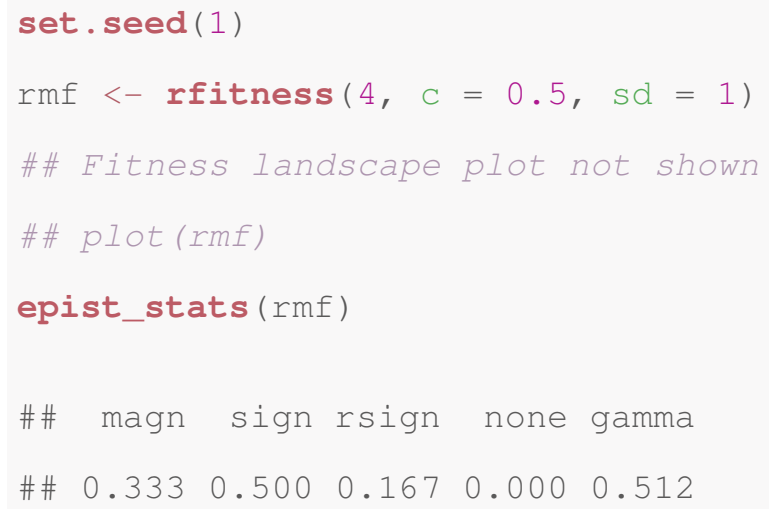


Finally, among the models provided by OncoSimulR, the NK or LK model is Kauffman's model [4, 14, 20, 21, 35]: for a genotype of $N$ loci, each locus has epistatic interactions with $K$ other loci; the log-fitness of a genotype is the sum of the contributions of all its loci, where the contributions of each locus to the $(\log )$ fitness are random deviates from some probability distribution (here a uniform $(0,1)$ ) that differ depending on the state of the given locus and all its $K$ interacting loci. Thus, we go from minimal epistasis when $K=1$ to maximal epistasis when $K=N-1$ (which is equivalent to a House of Cards model [14]).

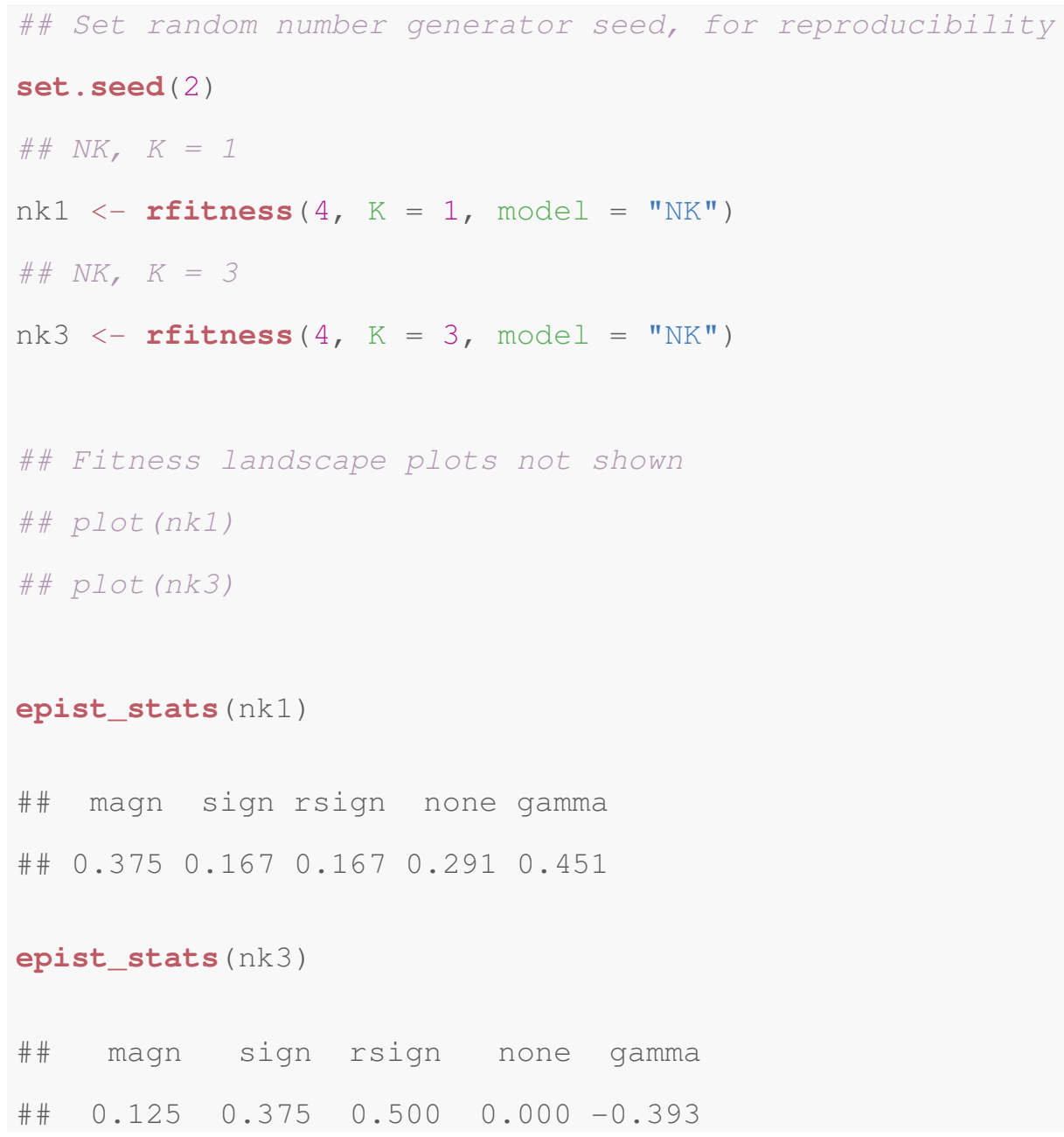

2.2.3 Specifying Epistasis Directly: Genes Without Interactions (No Epistasis)
To better understand Subheadings 2.2.4-2.2.7, we first provide a simple baseline example in which we specify a model without epistatic interactions: 


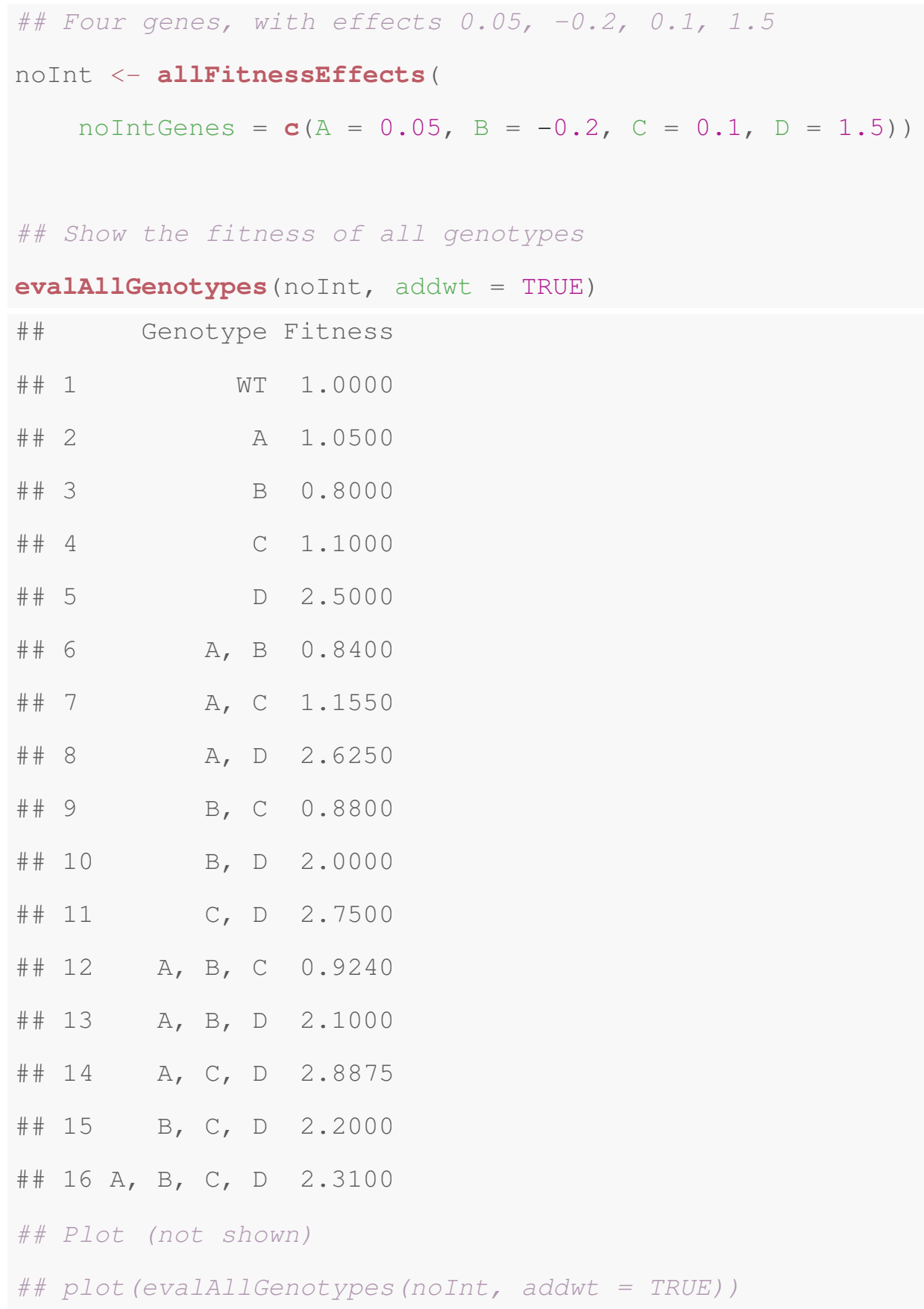

Computing epistasis statistics in these cases requires using the log of fitness, since we are using a multiplicative fitness specification: we specify the fitness effects of mutations using a multiplicative model-see also Subheading 2.3.1-, $\prod\left(1+s_{i}\right)$, where $s_{i}$ is the fitness effect of gene or gene interaction $i$ (thus we are explicitly modeling the effects of genes and gene interactions). Before we compute the epistasis specifications, however, we will check that fitness is what it should be under a multiplicative model for the contributions of genes (which is additive in the log scale): 
Now, compute epistasis statistics, first incorrectly setting use_log = FALSE:

all (evalAllGenotypes (noInt, addwt = TRUE) [, "Fitness"] == c $(1$,

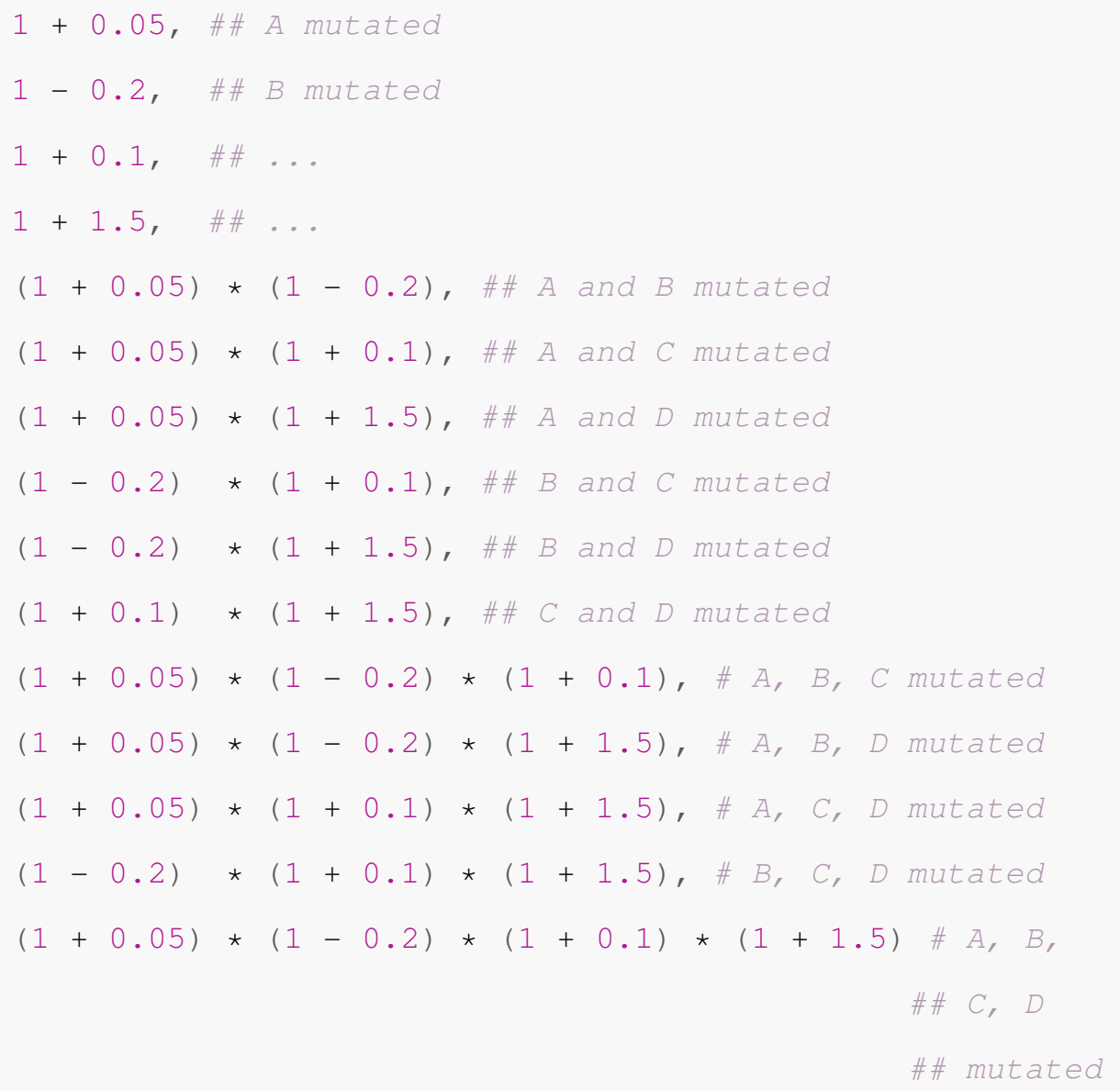

\#\# [1] TRUE

Now, set use_log = TRUE, to correctly compute the epistasis statistics, which shows there is no epistasis as all genes contribute additively in the log scale:

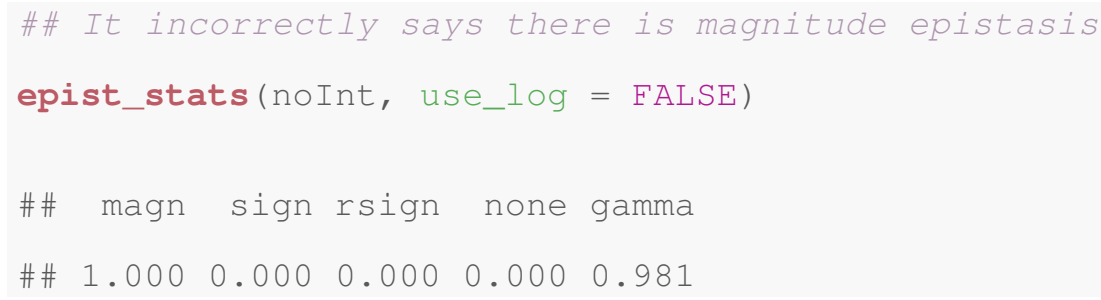




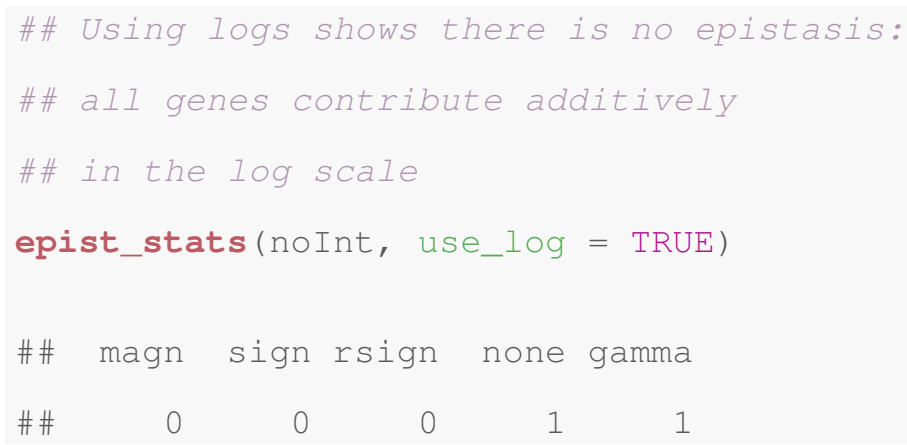

2.2.4 Specifying Epistasis Directly: Two Alternative Specifications of Epistasis

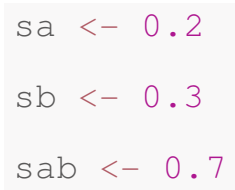
as shown in Table 1.

To make the example concrete, let $s_{a}=0.2, s_{b}=0.3, s_{a b}=0.7$. We specify the above scenario as follows: 


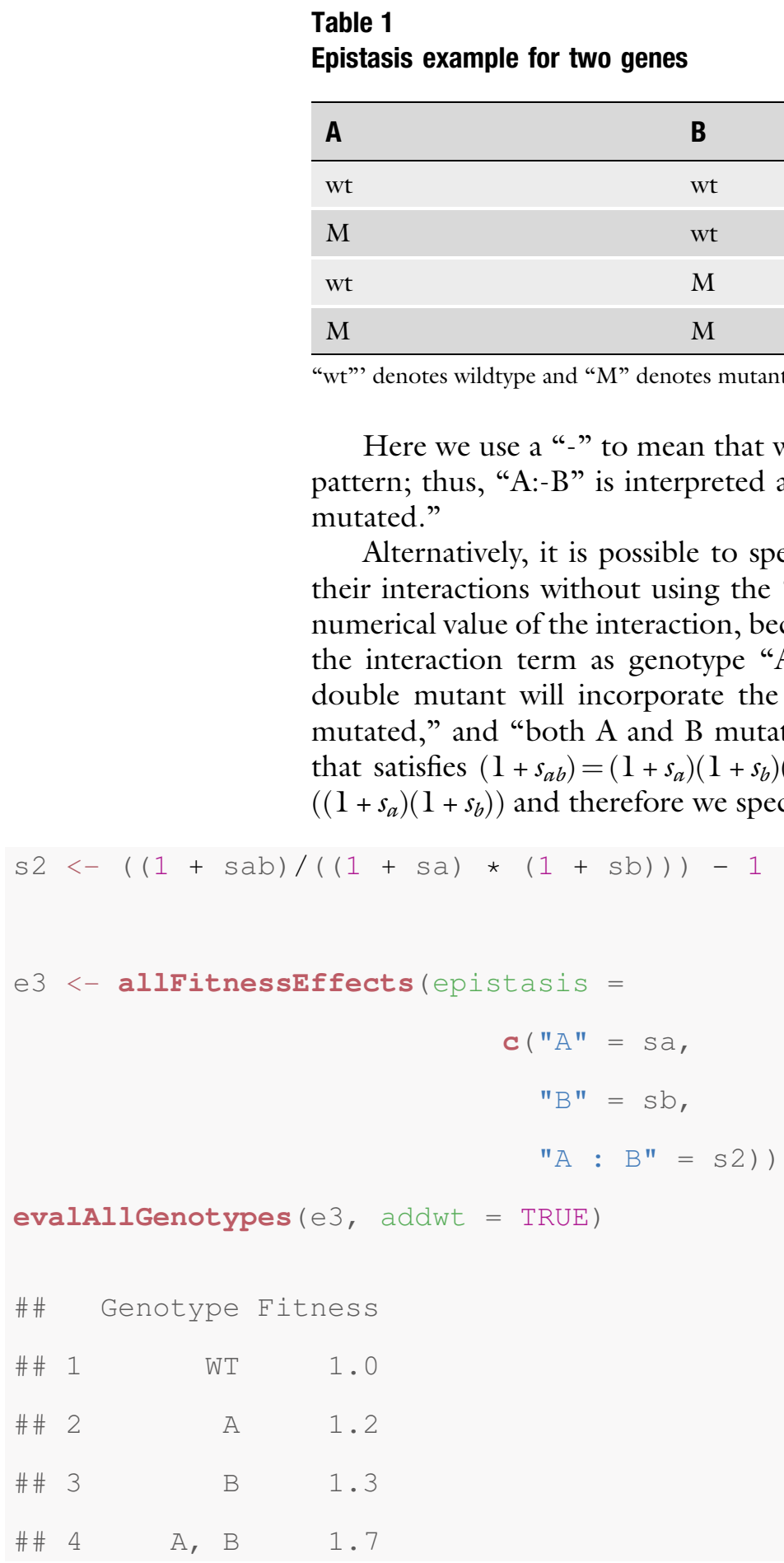

For example, this is the way you would specify epistasis with FFPopSim [37]. Whether this specification or the previous one with "-" is simpler will depend on the model. For synthetic mortality and viability (see below, Subheadings 2.2.6 and 2.2.7), using "-" makes it simpler to map genotype tables to fitness effects. 
Estimates of epistasis are the same regardless of how we specify the model (and note we continue using use_log = TRUE to obtain the epistasis statistics):

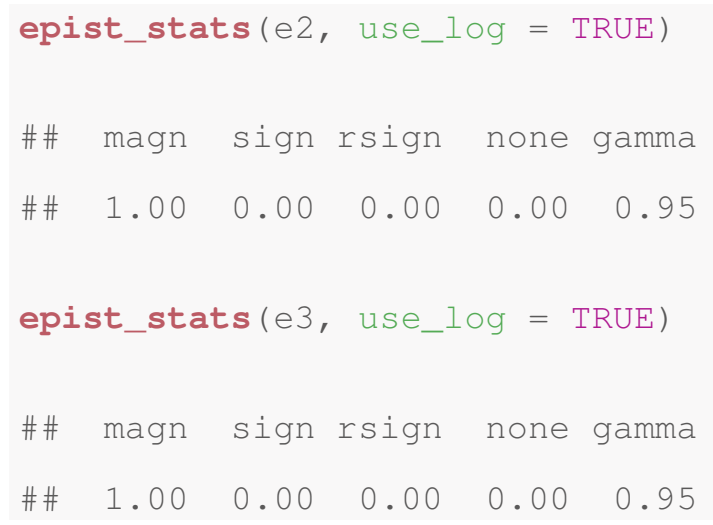

2.2.5 Two Alternative Specifications of Epistasis: A Three-Gene Example
To further illustrate the two mechanisms for epistasis specification, here we show a more complex three-gene example. We want to use the epistatic interactions where there is no epistasis between genes $\mathrm{A}$ and $\mathrm{C}$, but there is epistasis between genes $\mathrm{A}$ and $\mathrm{B}$, and between genes $\mathrm{B}$ and $\mathrm{C}$, as shown in Table 2 .

We can specify that model, providing numerical values for each $s$, as follows:

\section{Table 2}

\section{A three-gene fitness specification with epistasis}

\begin{tabular}{|c|c|c|c|}
\hline A & B & C & Fitness \\
\hline wt & wt & wt & 1 \\
\hline $\mathrm{M}$ & wt & wt & $1+s_{a}$ \\
\hline wt & M & wt & $1+s_{b}$ \\
\hline wt & wt & $\mathrm{M}$ & $1+s_{c}$ \\
\hline M & M & wt & $1+s_{a b}$ \\
\hline wt & M & M & $1+s_{b c}$ \\
\hline M & wt & M & $\left(1+s_{a}\right)\left(1+s_{c}\right)$ \\
\hline M & M & M & $1+s_{a b c}$ \\
\hline
\end{tabular}

"wt" denotes wildtype and " $M$ " denotes mutant. Note that the mutant for exactly A and $\mathrm{C}$ has a fitness that is the product of the individual terms (so there is no epistasis in that case) 


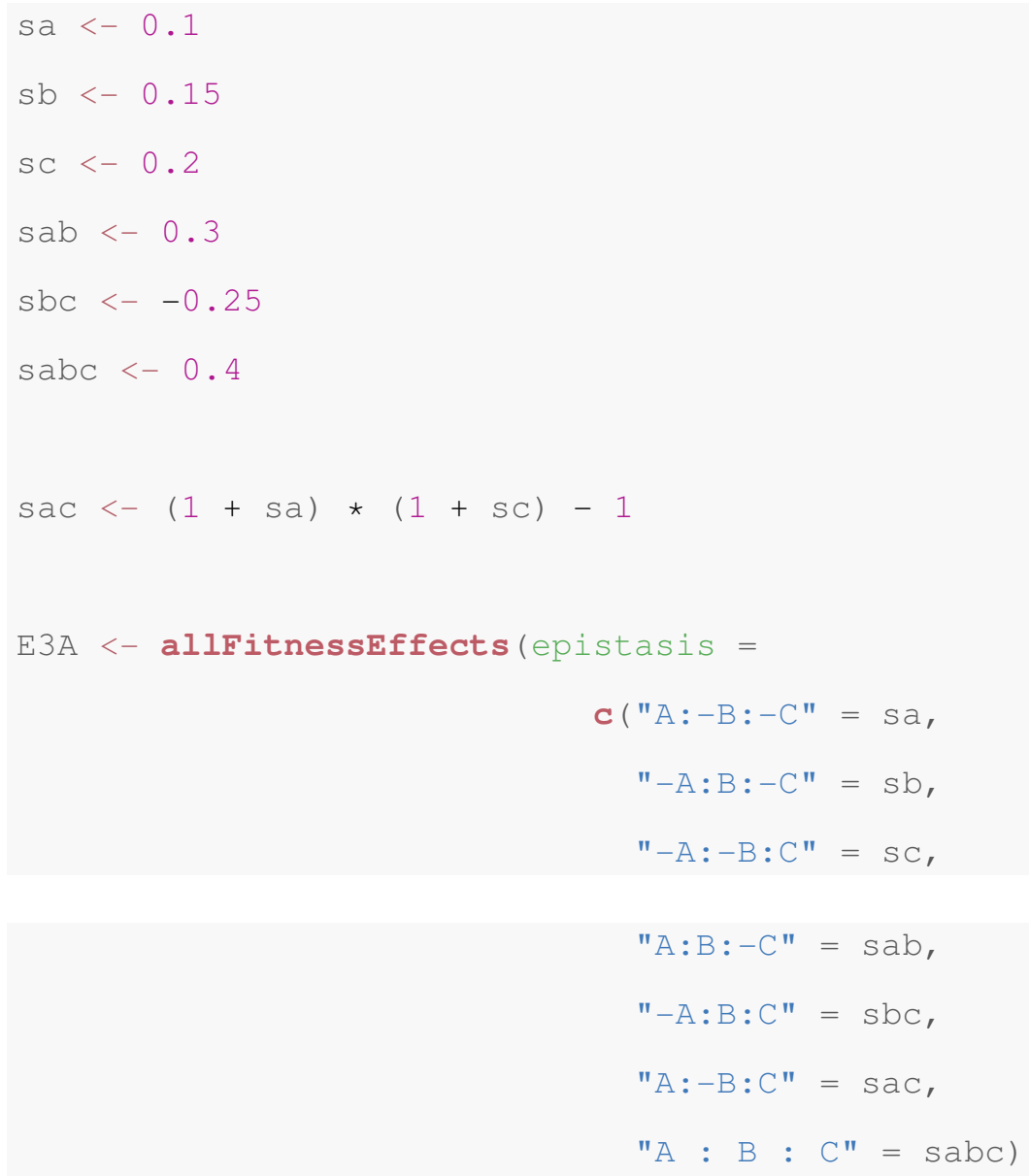


We needed to pass the $s_{a c}$ coefficient explicitly, even if that term is the product of the corresponding terms for the individual loci, because a full specification is required when using the "-".

We can use the alternative specification without "_", but we will need to perform some calculations to obtain some of the coefficients under this parameterization. To make it easier to tell the differences from the previous specification, I use capital " $S$ " in what follows where the numerical values differ from the previous specification. Note that we can avoid specifying "A:C", as it just follows from the individual " $\mathrm{A}$ " and " $\mathrm{C}$ " terms, but we need to obtain new $S_{a b}, S_{b c}, S_{a b c}$ :

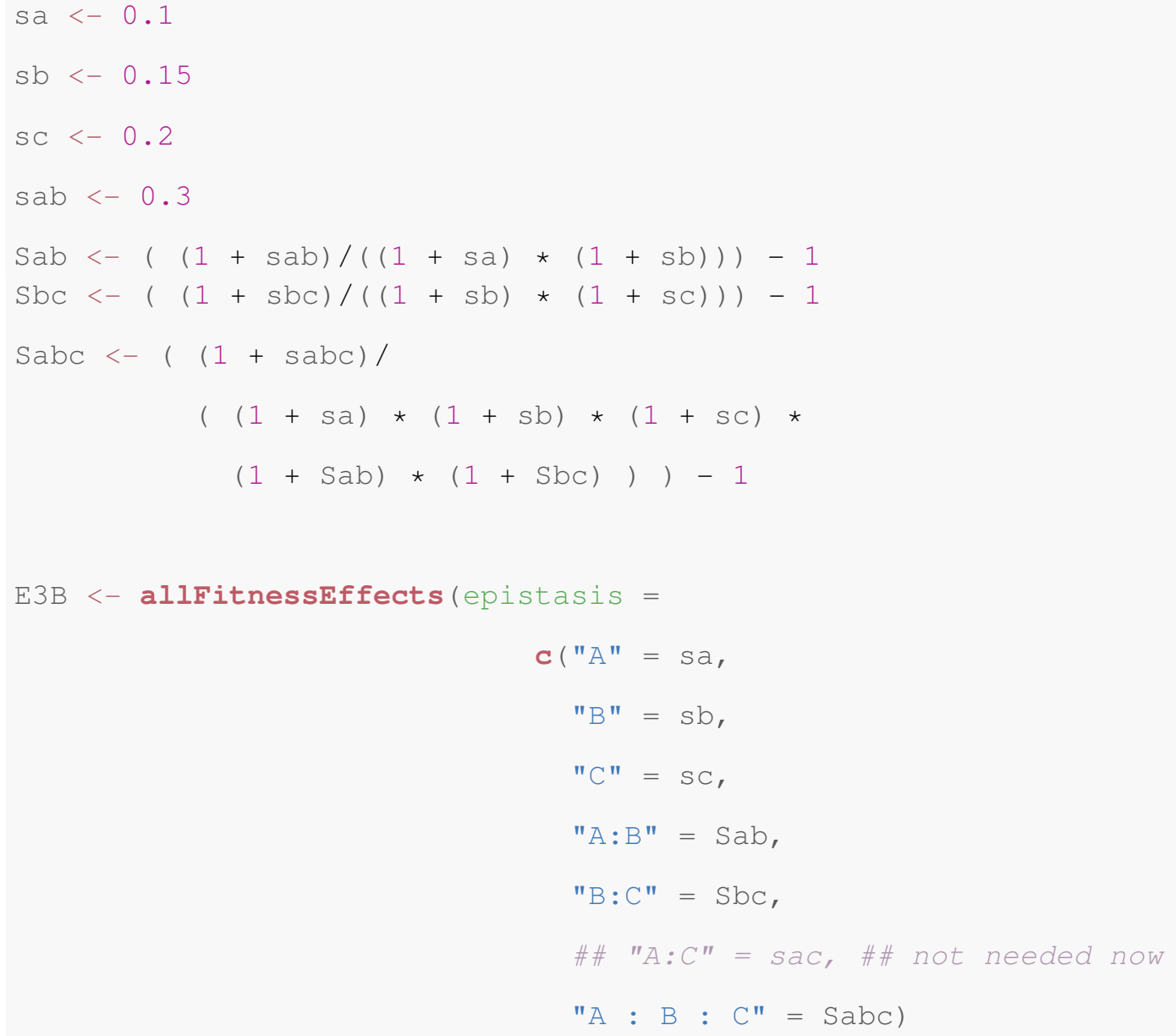




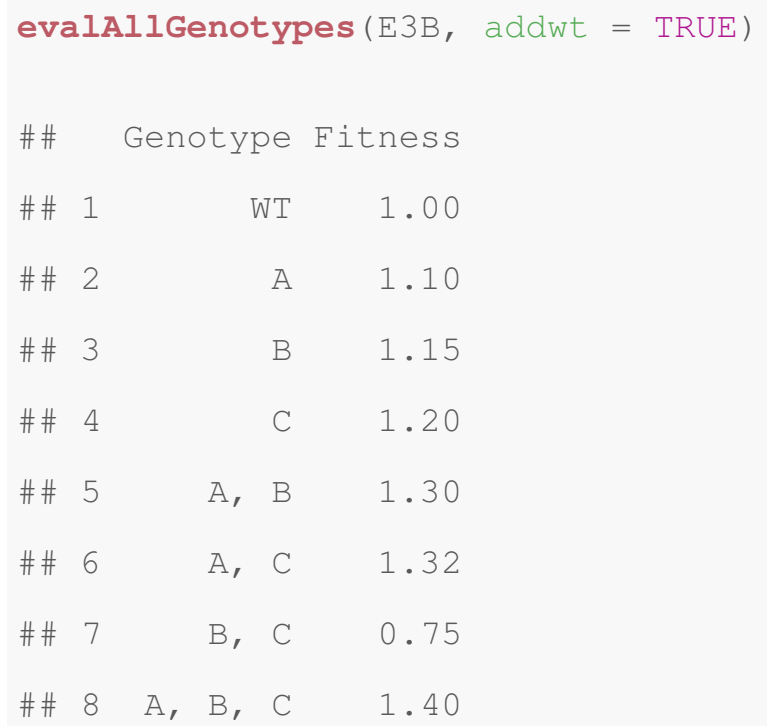

The actual fitness is the same:

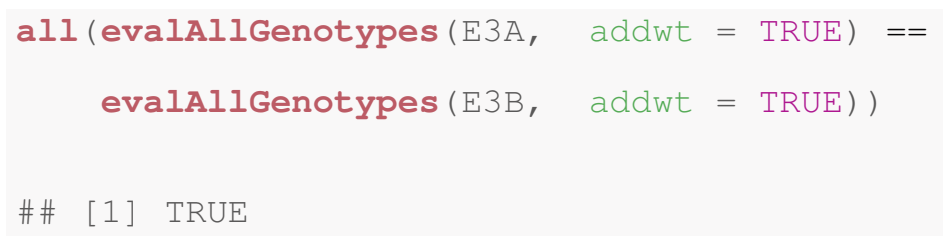

Epistasis statistics are the same:

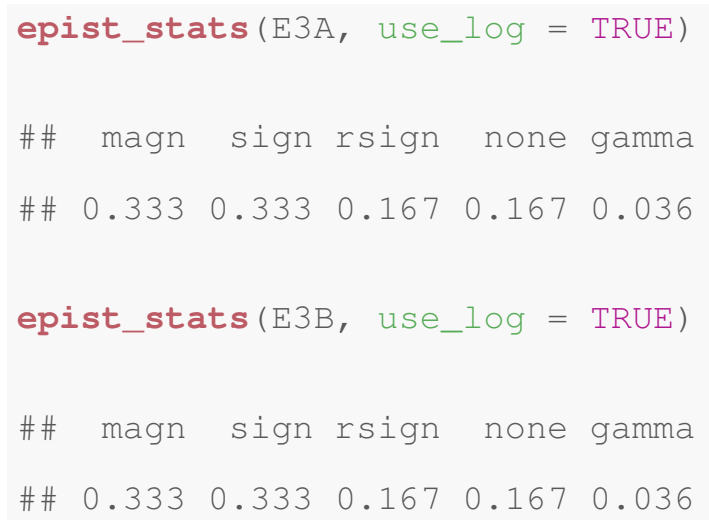

We can check the output from the above epistasis calculations using the graphical procedure for determining magnitude, sign, and reciprocal sign epistasis described in $[7,14]$. The two cases with magnitude epistasis (frequency of $2 / 6$ ) correspond to the sets "abc", "aBc", "Abc", "ABc" on the one hand and "Abc", "AbC", 


\section{Table 3}

A simple synthetic viability example

\begin{tabular}{lll}
\hline A & B & Fitness \\
\hline wt & wt & 1 \\
M & wt & $1+s_{a}$ \\
wt & $M$ & $1+s_{b}$ \\
M & $M$ & $1+s$ \\
\hline
\end{tabular}

"wt"' denotes wildtype and "M" denotes mutant. $s>0, s_{a}<0, s_{b}<0$

"ABc", "ABC" on the other (where a capital letter denotes that the given locus is mutated, e.g., "AbC" denotes the genotype with both $\mathrm{A}$ and $\mathrm{C}$ mutated). The two cases with sign epistasis correspond to the two sets "abC", "aBC", "AbC", "ABC" and "aBc", "ABC", "ABc", "ABC". The case with reciprocal sign epistasis (frequency of $1 / 6$ ) to the set "abc", “aBc", "abC", “aBC". Finally, the value for "none" (frequency of $1 / 6$ ) corresponds to the set of four genotypes "abc", "Abc", "abC", and "AbC" .

Synthetic viability, where each individual mutant is lethal or has decreased fitness but the double mutant is viable, is just a case of reciprocal sign epistasis, but we illustrate it here separately with a minimal example. Suppose we want to model fitness as shown in Table 3.

We will set $s=0.2$, and $s_{a}=s_{b}=-0.5$. Then, we can specify fitness as follows:

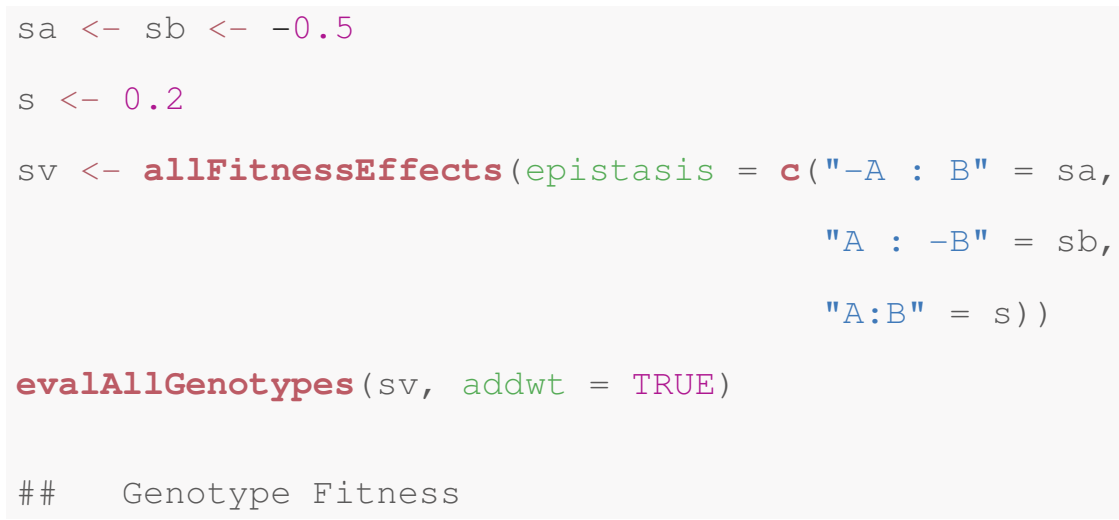




$\begin{array}{lrrrr}\text { \#\# } & 1 & & \text { WT } & 1.0 \\ \text { \#\# } & 2 & & \text { A } & 0.5 \\ \text { \#\# } & 3 & & \text { B } & 0.5 \\ \text { \#\# } & 4 & \text { A, } & \text { B } & 1.2\end{array}$

epist_stats (sv, use_log = TRUE)
\# magn sign rsign none gamma
$\begin{array}{lllllll}\# & 0.000 & 0.000 & 1.000 & 0.000 & -0.973\end{array}$

2.2.7 Synthetic Sickness, Synthetic sickness and synthetic lethality or synthetic mortality are Synthetic Lethality or Synthetic Mortality another case of reciprocal sign epistasis. Here, each single mutant leads to an increase in fitness but the double mutant leads to a decrease in fitness, including possible non-viability of the double mutant (synthetic lethality or synthetic mortality). A very simple case is shown in Table 4.

We will make $s_{a}=0.1, s_{b}=0.2, s_{a b}=-0.8$. We can specify it as

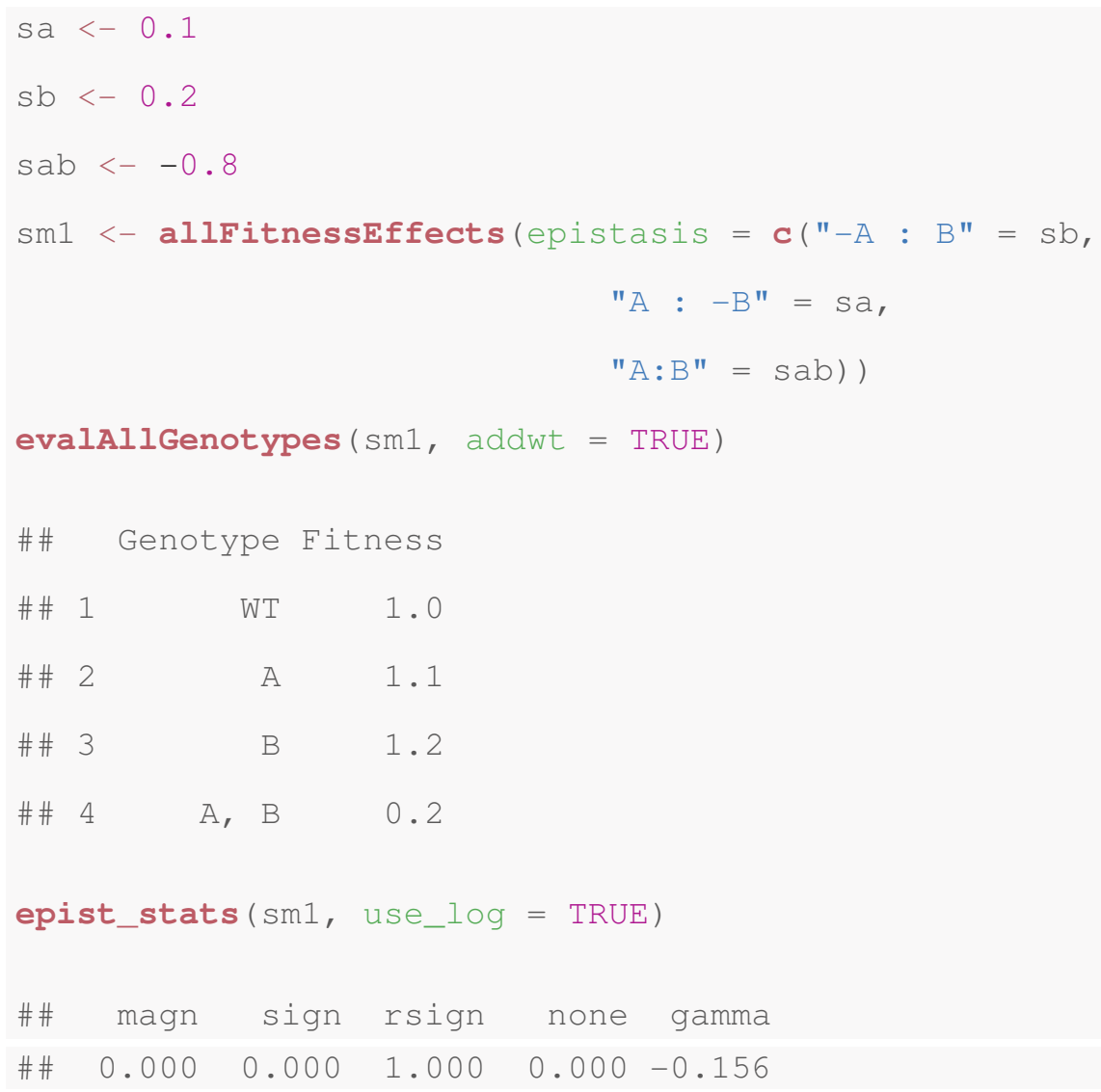


Table 4

A simple synthetic sickness example

\subsection{Simulating Evolution}

\subsubsection{Growth Models}

\begin{tabular}{lll}
\hline A & B & Fitness \\
\hline $\mathrm{wt}$ & $\mathrm{wt}$ & $\mathrm{l}$ \\
$\mathrm{M}$ & $\mathrm{wt}$ & $1+s_{a}$ \\
$\mathrm{wt}$ & $\mathrm{M}$ & $1+s_{b}$ \\
$\mathrm{M}$ & $\mathrm{M}$ & $1+s_{a b}$ \\
\hline
\end{tabular}

"wt" denotes wildtype and "M" denotes mutant. $s_{a}>0, s_{b}>0, s_{a b}<0$

The main function for simulating evolution with OncoSimulR is oncosimulIndiv. In addition, functions oncosimulpop and oncosimulsample allow us to run multiple simulations and in particular oncosimulPop allows us to use multiple chores to parallelize execution.

Once epistasis or, equivalently, fitness of genotypes has been specified, as explained above (Subheading 2.2), we can simulate evolution using any of the oncosimul* functions. Before actually running simulations (Subheading 2.3.3), though, we need to decide about the growth model and mutation rates of genes.

OncoSimulR uses a continuous time model. The main choice for growth models is between a model with exponential growth or a model with carrying capacity (the model of McFarland et al. [2426]) (but see also Note 4 ). In both cases, when we specify the fitness effects of genes and gene interactions, and as shown above (Subheadings 2.2.4-2.2.7), we evaluate fitness using the usual $[2,8,18$, 37] multiplicative model: fitness is $\prod\left(1+s_{i}\right)$ where $s_{i}$ is the fitness effect of gene (or gene interaction) $i$. In both models this fitness refers to the growth rate. The original model of McFarland et al. [26] has a slightly different parameterization, but you can go easily from one to the other (see below, "Birth rate parameterization in the model with carrying capacity"). If you specify fitness of genotypes directly (Subheadings 2.2.1 and 2.2.2), then that is also taken as the birth rate of genotypes.

In the model with exponential growth we specify the growth rate, fixing death rate at 1 (it is possible to modify this if really needed, but there is rarely any need to do so). The model with carrying capacity follows the model of McFarland et al. [24-26]: mutations affect the birth rate, with the death rate being density dependent (see below).

In OncoSimulR, we choose the exponential growth model setting model $=$ "Exp" in the call to functions oncoSimul*. The model with carrying capacity is specified using model $=$ "MCFL". Note that even if the MCFL shows density dependence, there is no frequency-dependence of fitness in any of the models (but see Note 5 ). 
2.3.2 Mutation Rates, Mutator Genes
Death Rate in the Model with Carrying Capacity: For death rate, we use the expression that McFarland et al. [26, see p. 2911] use " $(.$.$) for large cancers (grown to 10^{6}$ cells)": $D(N)=\log (1+N / K)$ where $K$ is the initial equilibrium population size. As the authors explain, for large $N / K$ the above expression "(...) recapitulates Gompertzian dynamics observed experimentally for large tumors." By default, OncoSimulR uses a value of $K=$ initSize $/\left(e^{1}-1\right)$ so that the starting population (which starts with population size $=$ initSize) is at equilibrium.

Birth Rate Parameterization in the Model with Carrying Capacity: For the birth rate, in the original model in McFarland et al. [26], the effects of drivers contribute to the numerator of the birth rate, and those of the (deleterious) passengers to the denominator as: $\frac{(1+s)^{d}}{\left(1+s_{p}\right)^{2}}$, where $d$ and $p$ are, respectively, the total number of drivers and passengers in a genotype, and the fitness effects of all drivers are the same $(s)$ and that of all passengers the same $\left(s_{p}\right)$. Note that as written above and as explicitly mentioned in McFarland et al. ( [26] p. 2911, and [24], p. 9) “(...) $s_{p}$ is the fitness disadvantage conferred by a passenger." In other words, the larger the $s_{p}$, the more deleterious the passenger. As explained above, however, we use a multiplicative model $\prod\left(1+s_{i}\right)$, where genes and their interactions can have arbitrary positive or negative effects (as we saw in Subheadings 2.2.4-2.2.7). Thus, if one is given a model specified as in the parameterization $\frac{(1+s)^{d}}{\left(1+s_{p}\right)^{p}}$ one would simply need to rewrite the appropriate term for the $s_{p}$ as $\left(1+s_{i}\right)=-s_{p} /\left(1+s_{p}\right)$, for the $s_{i}$ in our parameterization.

OncoSimulR can use both a common mutation rate for all loci as well as locus-specific mutation rates. Below we show two calls to oncosimulIndiv, the first one, rmf_common, with a common mutation rate of $1 e-7$ for all loci, and the second, rmf_loci_spec, with different mutation rates for each locus. We reuse the RMF fitness specification from Subheading 2.2.2.

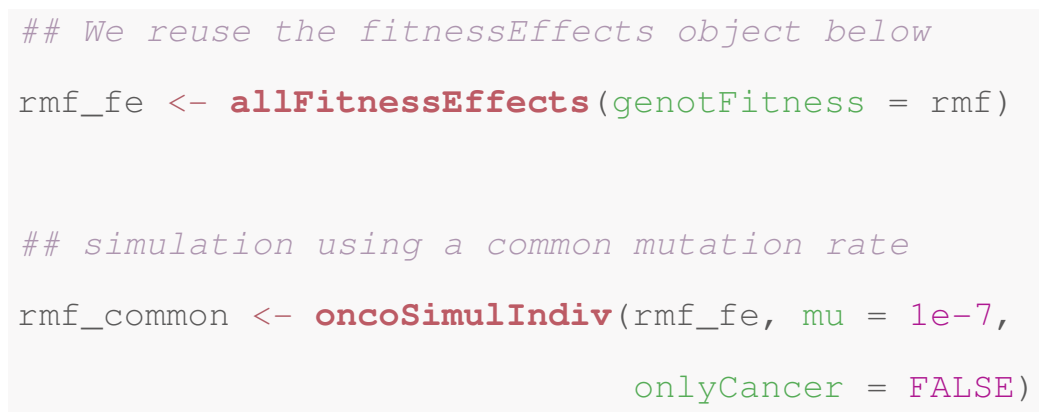




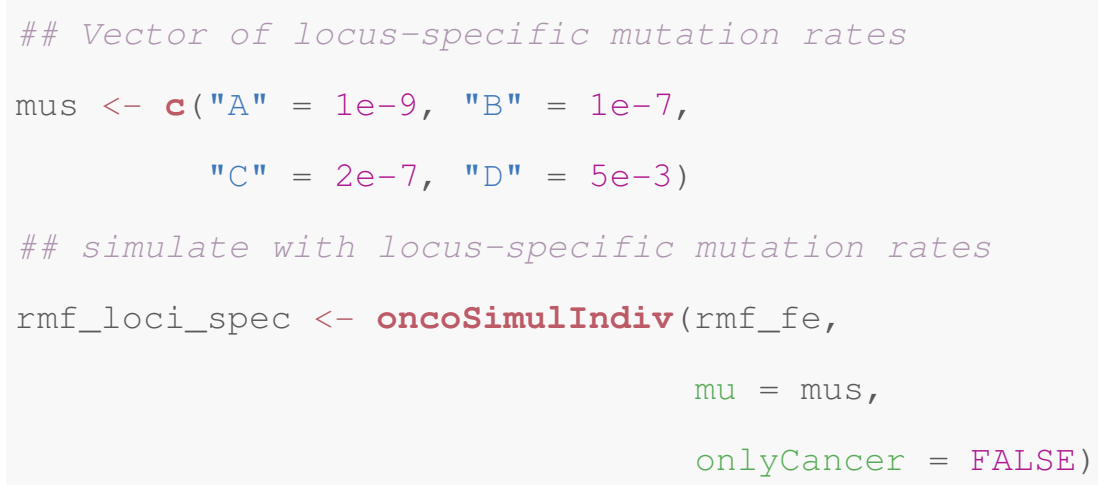

It is also possible to specify mutator/antimutator genes (e.g., $[15,36])$; these genes, when mutated, lead to an increase/ decrease in the mutation rate across the genome. Mutator/antimutator genes must be a subset of the genes in the fitness effects (if you want to use mutator genes that have no direct fitness effects, give them a fitness effect of 0 - see examples in the documentation). In the example below, we specify that mutating gene "A" leads to an increase by a factor of fifty of the mutation rate. See also Note 6 for numerical issues that can result from using multiple mutator genes.

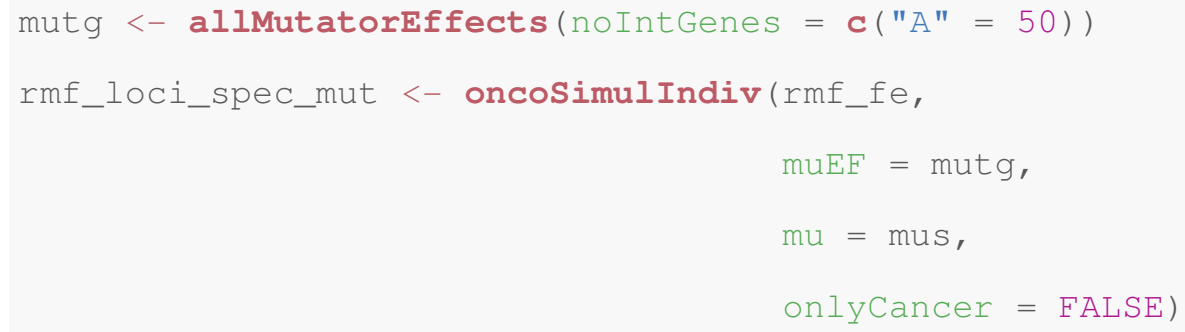

2.3.3 Running

Simulations Until

Pre-specified Conditions

Are Met
In addition to the growth model, fitness effects, and possible mutator effects and locus-specific mutation rates, you need to decide:

- Where simulations will start from. This involves deciding the initial population size (argument initsize); sometimes you might want to start the simulations from a specific genotype (see Note 7).

- When will simulations stop: how long to run simulations, and whether or not to require simulations to reach a particular condition. This is covered below.

OncoSimulR provides very flexible ways to decide when to stop a simulation: 
- Using option onlycancer $=$ TRUE.

A simulation will be repeated until any one of the conditions below is met, if this happens before the simulation reaches finalTime. Because OncoSimulR was originally developed to simulate cancer evolution, this is often referred as "reaching cancer" but we can refer to it as "reach whatever interests me." These conditions are:

- Total population size becomes larger than detectionsize.

- A gene, gene combination, or genotype among those listed in fixation becomes fixed in the population (i.e., has a frequency of 1 or very close to 1 ); see Subheadings 2.3.4-2.3.6.

- The tumor is detected according to a stochastic detection mechanism, where the probability of "detecting the tumor" increases with population size; see Subheading 2.3.7.

- The number of drivers in any one genotype becomes equal to, or larger than, detectionDrivers (this could also be used to stop the simulation as soon as a specific genotype is found, by using the genes that make that genotype as the drivers, but the mechanism in Subheading 2.3.5 is generally simpler). This option is only reasonable in scenarios where we want to differentiate between driver and passenger genes, and it requires specifying driver genes; this will not be further discussed here.

The simulations exit as soon as any of the exiting conditions is reached; therefore, if you only care about one condition, set the other conditions to NA.

- onlyCancer $=$ FALSE.

A simulation will run only once, and will exit as soon as any of the above conditions are met or as soon as the total population size becomes zero or we reach $f$ inalTime.

Using onlycancer = FALSE will often be the setting you want to use to examine general population genetics scenarios without focusing on possible sampling issues; set finaltime to the value you want and set onlycancer = FALSE; in addition, set detectionProb to "NA" and detectionDrivers and detectionsize to "NA" or to huge numbers. This way you simply collect the simulation output at the end of the run, regardless of what happened with the population (it became extinct, it did not reach a large size, it did not accumulate drivers, etc.).

Under the onlycancer $=$ TRUE case, if we reach finalTime (or the population size becomes zero) before any of the "reach cancer" conditions have been fulfilled, the simulation will be repeated again, within the limits given by the following parameters 
2.3.4 Fixation of Genes and Gene Combinations to the functions oncosimulindiv or oncosimulpop: max. wall.time: the total wall time we allow an individual simulation to run; max. num.tries: the maximum number of times we allow a simulation to be repeated to reach cancer; if you use oncosimulsample, max.wall.time.total and max.num.tries. total, similar to the previous two, but specific for function oncoSimulsample, are also of application. If the specified conditions for "reaching cancer" cannot be met, no object with the population state (genotypes and population sizes) will be returned (i.e., simulations will abort without returning the population state, as no simulation has achieved the specified conditions).

Simulations will exit when any of the genes or gene combinations in the vector (or list) fixation, passed to the oncosimul* functions, reaches a frequency of 1 , or very close to 1 (see Subheading 2.3.6). The gene combinations might share genes (i.e., might have non-zero intersection). As explained above, if we want simulations to only exit when fixation of those genes/gene combinations is reached, we will set all other stopping conditions to NA. Note that if the stopping conditions can never be reached, simulations will eventually abort (e.g., when max.wall.time or max.num. tries are reached). Since we are running simulations until fixation of genes, the Exp model will rarely be appropriate here: the MCFL model, that includes competition, is more appropriate.

The following code shows an example based in the model in Ochs and Desai [29]; the authors present a model like the one shown in Fig. 2 (the numerical values are arbitrarily set by me). In this model $s_{u}>0, s_{v}>s_{u}, s_{i}<0$ and we can only arrive at $v$ from $i$. Mutants "ui" and "uv" can never appear as their fitness is 0 , or $-\infty$, so $s_{u i}=s_{u v}=-1($ or $-\infty)$.

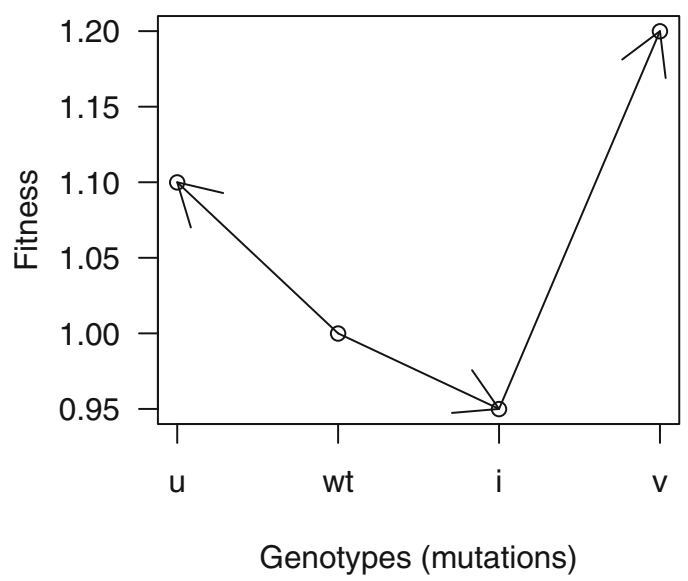

Fig. 2 Model from Ochs and Desai [29]. Actual numerical fitness values arbitrarily set by me to conform to their figure. Redrawn from Figure 1.a in [29] 
We can specify fitness by specifying epistatic effects:

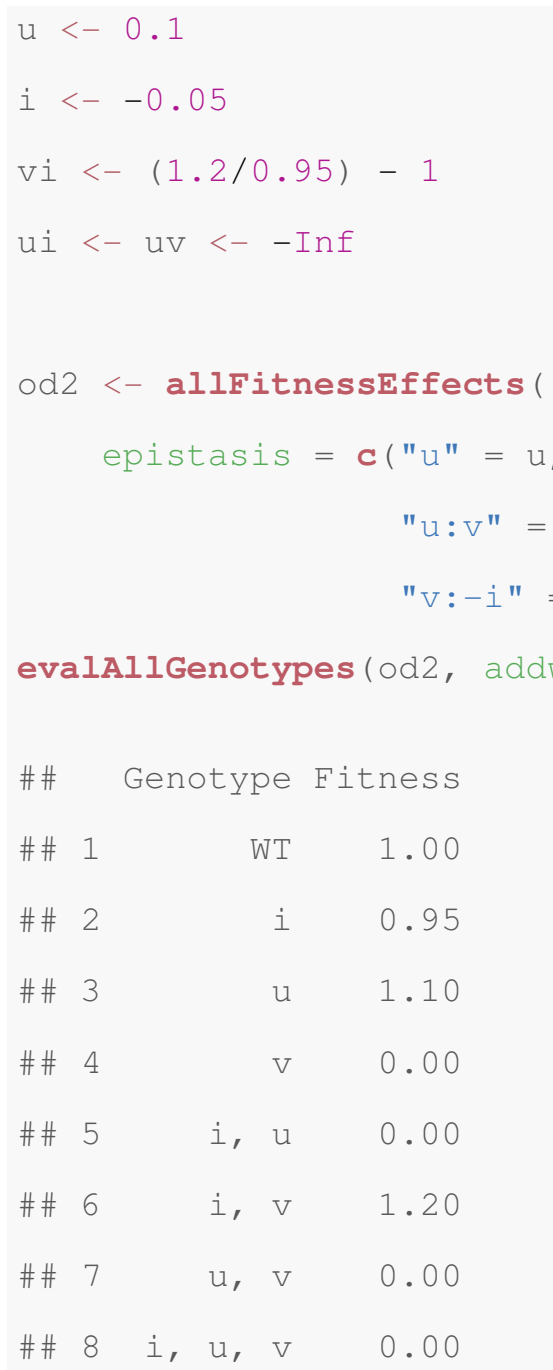

In p. 2, section "Simulations" of Ochs and Desai [29], they explain that "Each simulated population was evolved until either the uphill genotype or valley-crossing genotype fixed." To use the same procedure here, we specify that we want to end the simulation when either the "u" or the "v, i" genotypes have reached fixation, by passing those genotype combinations as the fixation argument (in this example using fixation $=c(" u "$, "v") would have been equivalent, since the " $v$ " genotype by itself has fitness of 0 ). Fixation will be the one and only condition for ending the simulations, and thus we set arguments detectionDrivers, finalTime, detectionsize, and detectionProb explicitly to NA. 
We want to run the simulations multiple times, so we use oncoSimulPop but we set the number of replicates to only 10 for the sake of speed in this example (a much larger number of replicates would be required for real cases):

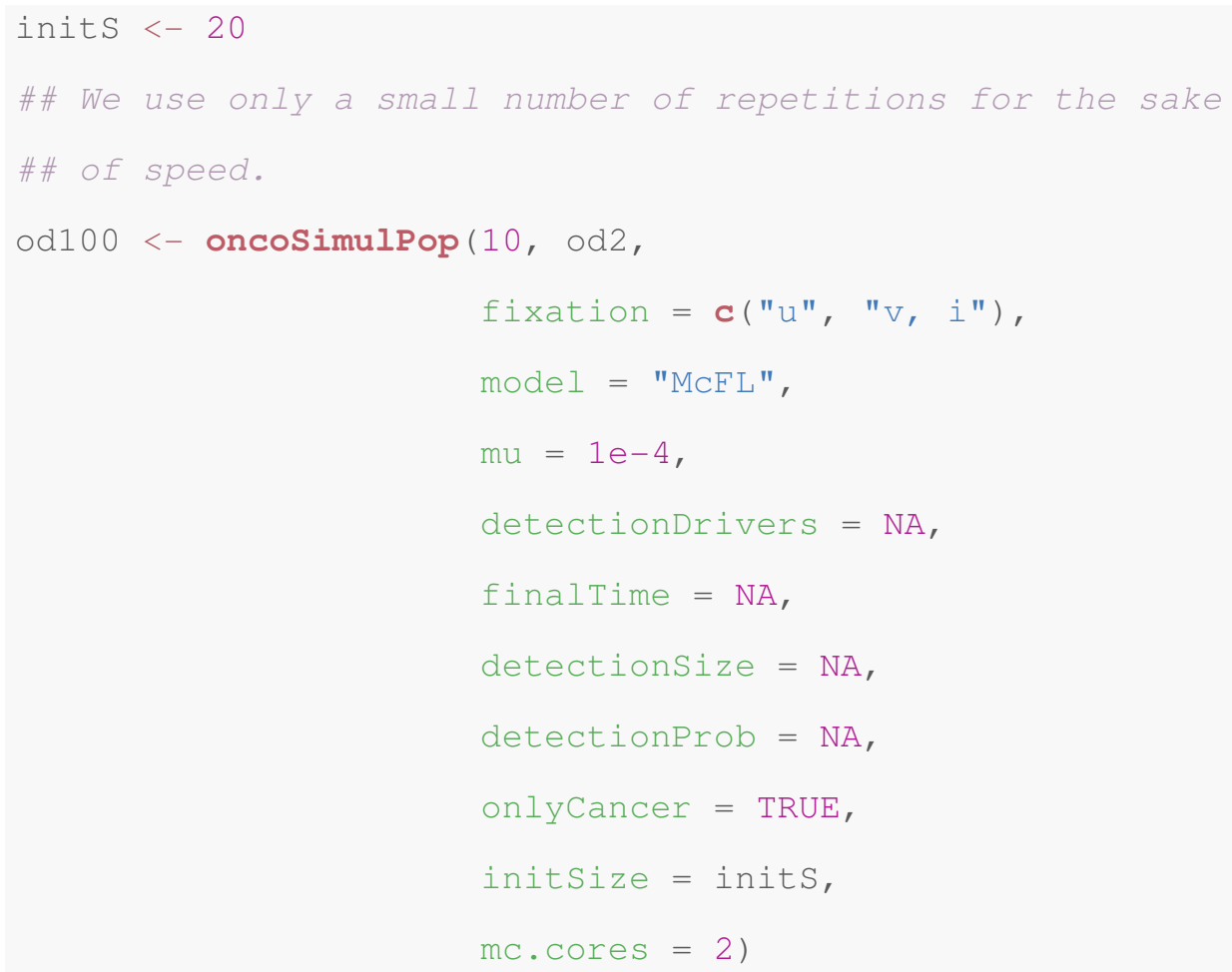

What is the frequency of each genotype among the simulations? (or, what is the frequency of fixation of each genotype?)

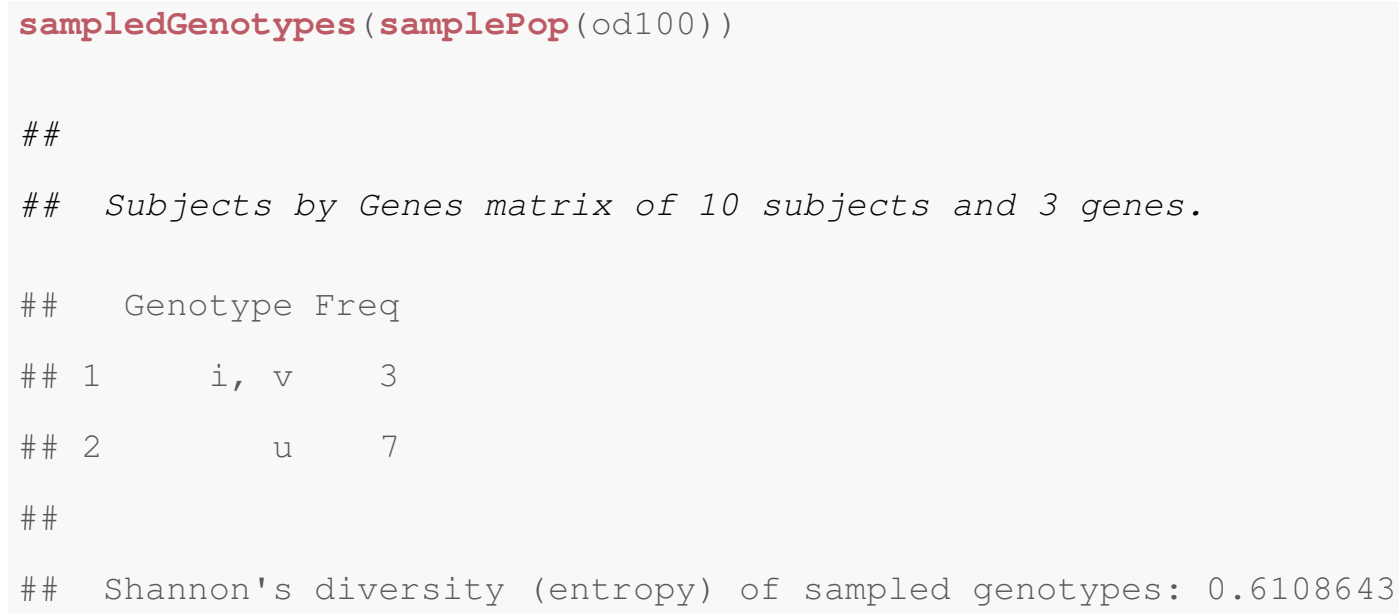

Note the variability in time to reach fixation 


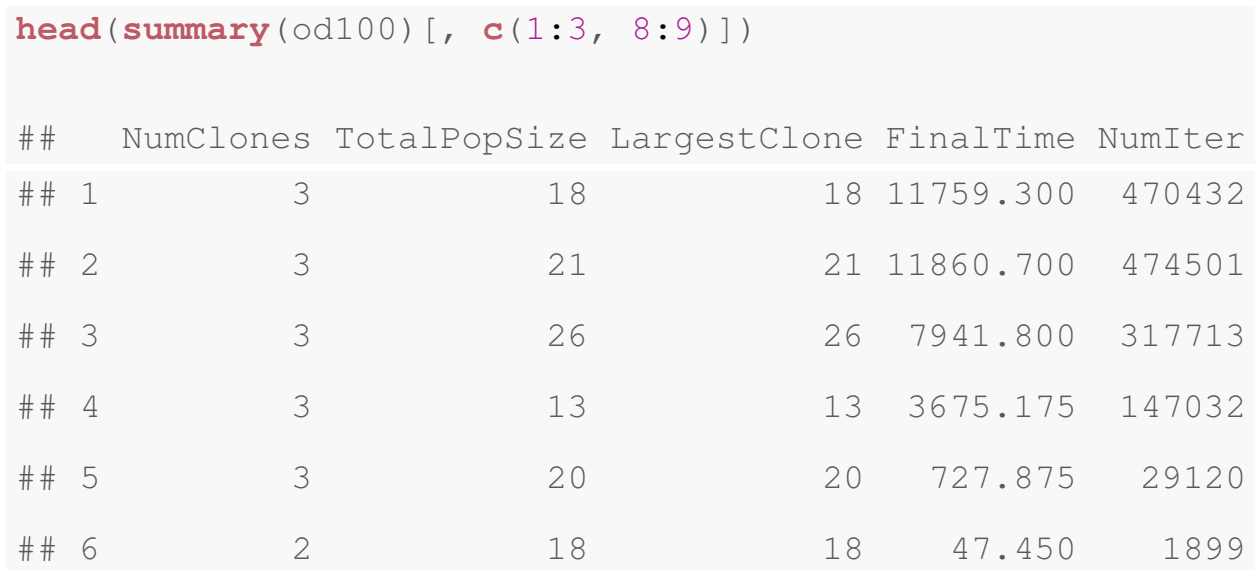

2.3.5 Fixation of Genotypes
Suppose you are dealing with a five loci genotype and suppose that you want to stop the simulations only if genotypes " $\mathrm{A}$ ", " $\mathrm{B}, \mathrm{E}$ ", or "A, B, C, D, E" reach fixation. You do not want to stop if, say, genotype "A, B, E" reaches fixation: the mechanism in Subheading 2.3.4 would not be useful here. To specify genotypes, you prepend the genotype combinations with a ",,", and that tells OncoSimulR that you want fixation of genotypes, not just gene combinations.

The following example illustrates the differences between the two mechanisms:

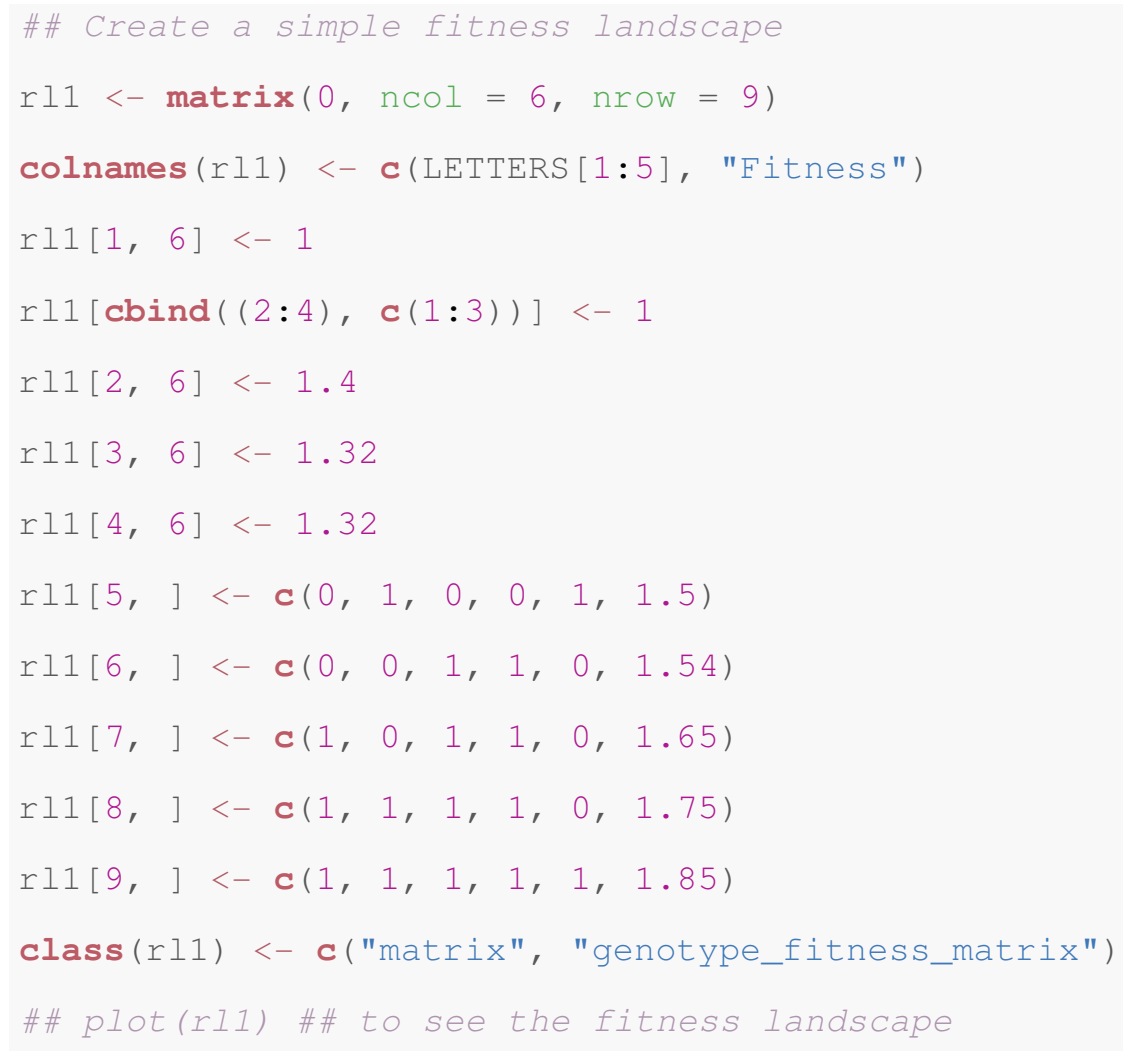




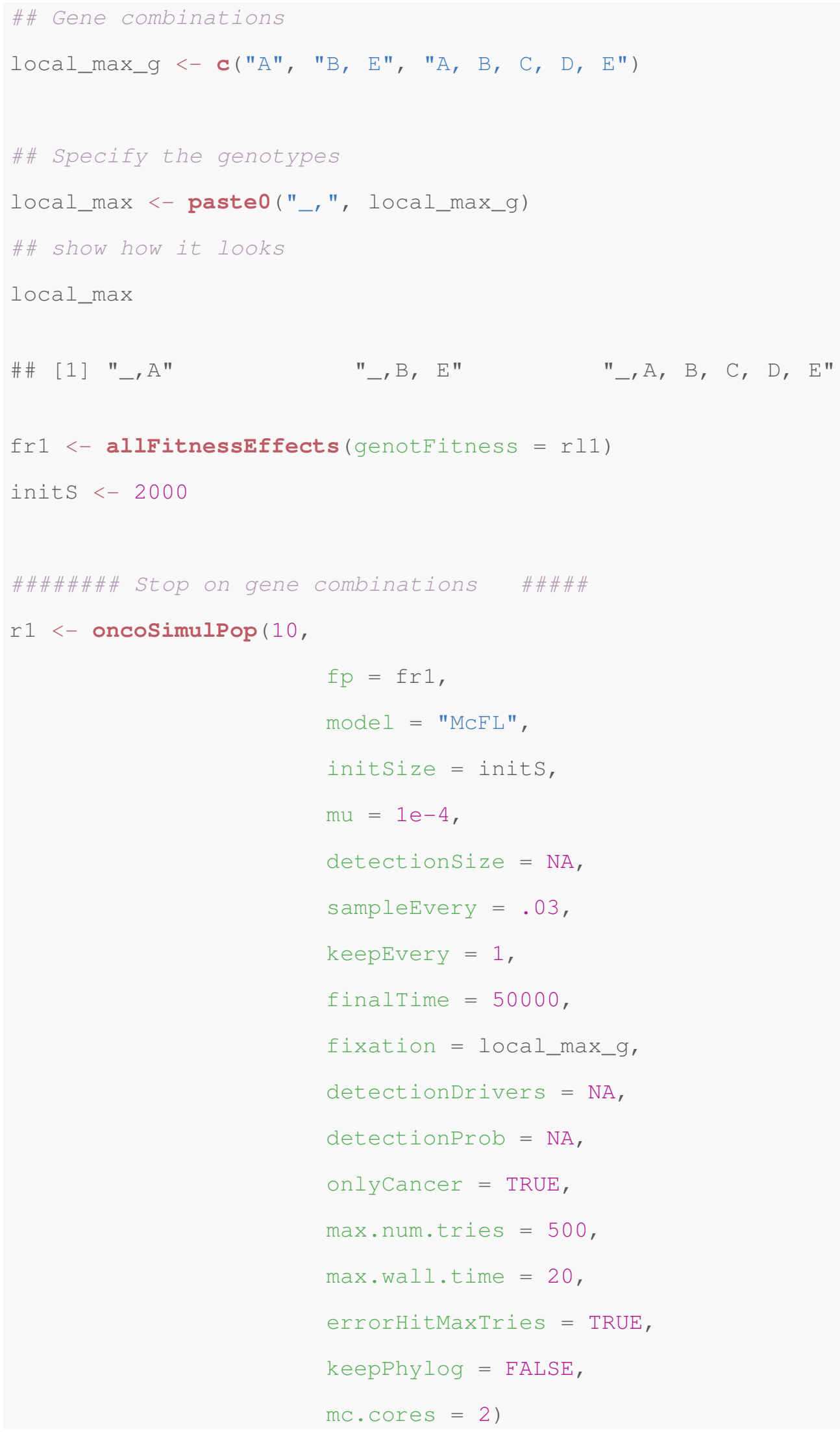


spl <- samplePop(r1, "last", "singleCell")

\# \#

\#\# Subjects by Genes matrix of 10 subjects and 5 genes.

\#\# Show the frequency of final composition of the populations

sampledGenotypes ( $\mathrm{sp} 1$ )

\#\# Genotype Freq

$\begin{array}{llll}\# \# & 1 & \text { A } & 6\end{array}$

\#\# 2 A, B, C, D 2

\#\# $3 \quad$ B $\quad E \quad 2$

\# \#

\#\# Shannon's diversity (entropy) of sampled genotypes: 0.9502705

\#\#\#\#\# Stop on genotypes \#\#\#\#

r2<- oncoSimulpop $(10$,

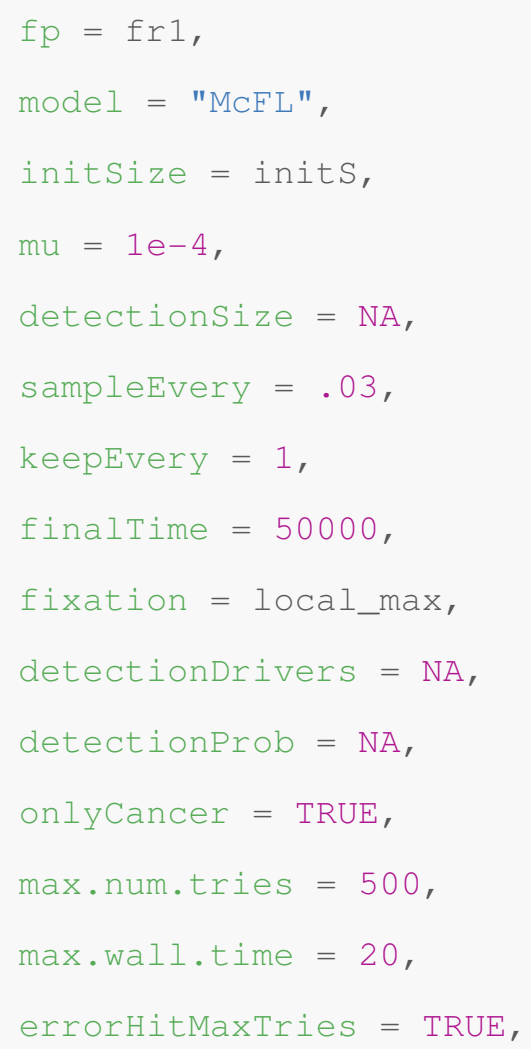


2.3.7 Stochastic Detection Mechanism fixation_min_size: you might only want to consider fixation to have happened if a minimal size has been reached; this can help eliminate local maxima that have fitness that is barely above that of the wildtype genotype. (The default is 0 .)

This process is controlled by the argument detectionProb. Under this process, we simulate stopping a simulation when a tumor is detected, where the probability of "tumor detection" increases with the total population size. The probability of detection is given by

$$
P(N)= \begin{cases}1-e^{-c((N-B) / B)} & \text { if } N>B \\ 0 & \text { if } N \leq B\end{cases}
$$

where $P(N)$ is the probability that a tumor with a population size $N$ will be detected, and $c$ (argument $c P D e t e c t$ in the oncosimul* functions) controls how fast $P(N)$ increases with increasing population size relative to a baseline, $B$ (PDBaseline in the oncosimul* functions). This function is evaluated at regularly spaced times during the simulation, and the decision to exit the simulation is made by comparing $P(N)$ against a random uniform number. Using this exiting mechanism is probably only appropriate for modeling diseases such as cancer and will not be further discussed here. See the vignette and documentation for details and examples.

\section{Output and Data Analysis}

The output from the simulation functions oncosimulindiv, oncosimulpop, and oncosimulsample are lists (see details in the documentation). These lists contain, among other components, the state of the population (genotypes and number of cells) at the time of stopping the simulation and, for oncosimulindiv and oncoSimulPop, all other previous sampling times. What users do with the output will be completely dependent on the research question. Some of the questions that can be addressed with the output from OncoSimulR include:

- Effects of sign epistasis in the probability and time to cross fitness valleys. We have provided small examples in Subheadings 2.3.4 and 2.3.5.

- The predictability of evolution in complex fitness landscapes, as shown in $[13,19]$.

- The effects of mutator/antimutator genes in reaching particular genotypes or population sizes. 
- Whether we can recover restrictions in the order of accumulation of mutations with different types of epistatic relationships, as shown in $[10,12]$.

Simple examples illustrating those scenarios are provided in the vignette of the OncoSimulR package.

\section{Notes: Potential Pitfalls and Troubleshooting}

1. It is also possible to specify epistasis using Directed Acyclic Graphs (DAGs) that represent order dependencies in the accumulation of mutations. This is equivalent to specifying sign epistasis [12], and it is used by "cancer progression models" such as Conjunctive Bayesian Networks (CBN) [16, 17, 27], oncogenetic trees (OT) $[9,34]$, CAncer PRogression Inference (CAPRI) [5, 31], or CAncer PRogression Extraction with Single Edges (CAPRESE) [30]. This usage, however, can only represent sign epistasis, or relaxations of sign epistasis; see the vignette of the OncoSimulR package for examples.

2. OncoSimulR also allows us to specify fitness not in terms of genes but in terms of modules. This can be useful in some scenarios as discussed in, for example, $[6,32]$. Each module is a set of genes (and the intersection between modules is the empty set). Modules, then, play the role of a "union operation" over sets of genes. There is no major conceptual difference relative to what has been shown in this chapter, but one also needs to specify which genes belong to each module. This specification of fitness can be useful when using DAGs (as discussed in Note 1), but rarely in other scenarios.

3 . It is also possible to use additive models where the contribution of each mutated allele $i$ to the $\log$-fitness is $s_{i}$, where $s_{i}$ is a random deviate from a Normal distribution with user-specified mean and standard deviation, and the log-fitness of a genotype is the sum of the contributions of each mutated allele. This can be obtained using model = Additive in function rfitness with versions of OncoSimulR 2.17.7 and higher.

4. It is also possible to use an exponential growth model with birth rate fixed to 1 , and where the fitness specification affects the death rate, a model inspired in [3]. Specification of fitness effects via their effects on death rates, however, often leads to numerical issues (see documentation and vignette), and is not discussed in this paper.

5. A branch of OncoSimulR, https://github.com/rdiaz02/ OncoSimul/tree/freq-dep-fitness, includes an implementation with frequency-dependent fitness. This implementation includes all the features mentioned here, but also allows users to make fitness depend on the frequency of other genotypes. 
We have not mentioned these features as they extend beyond the specification of epistasis and the software requires users to carry out manual installation of software, until a new $\mathrm{R}$ building toolchain becomes stable.

6. If we use several mutator genes with independent effects it is easy to run into computational problems. Suppose we specify five mutator genes, each with an effect of multiplying by 50 the mutation rate. The genotype with all those five genes mutated will have an increased mutation rate of $50^{5}=312500000$. If you set the mutation rate to the default of $1 e-6$ you will have a mutation rate of 312 which makes no sense (and leads to several numerical problems and an early warning from the software).

7. It is possible to start simulations from a specific genotype. This can be done using the initmutant argument to the oncoSimul* functions.

\section{Acknowledgements}

Four anonymous reviewers for comments that improved the ms.

Funding: Supported by BFU2015-67302-R (MINECO/FEDER, $E U)$ to $R D U$.

\section{References}

1. Aita T, Uchiyama H, Inaoka T, Nakajima $M$, Kokubo T, Husimi Y (2000) Analysis of a local fitness landscape with a model of the rough Mt. Fuji-type landscape: application to prolyl endopeptidase and thermolysin. Biopolymers 54(1):64-79. https://doi.org/10.1002/( SICI)1097-0282(200007)54:1\%3C64::AIDBIP70\%3E3.0.CO;2-R. https://onlinelibrary. wiley.com/doi/abs/10.1002/\%28SICI\% 291097-0282\%28200007\%2954\%3Al\% 3C64\%3A\%3AAID-BIP70\%3E3.0.CO\%3B2-R

2. Beerenwinkel N, Eriksson N, Sturmfels B (2007) Conjunctive Bayesian networks. Bernoulli 13(4):893-909. https://doi.org/10. 3150/07-BEJ6133. http://projecteuclid. org/euclid.bj/1194625594

3. Bozic I, Antal T, Ohtsuki H, Carter H, Kim D, Chen S, Karchin R, Kinzler KW, Vogelstein B, Nowak MA (2010) Accumulation of driver and passenger mutations during tumor progression. Proc Natl Acad Sci USA 107:18545-18550. https://doi.org/10. 1073/pnas.1010978107. http://www.ncbi. nlm.nih.gov/pubmed/20876136

4. Brouillet S, Annoni H, Ferretti L, Achaz G (2015) MAGELLAN: a tool to explore small fitness landscapes. bioRxiv, p. 031583. https:// doi.org/10.1101/031583. http://biorxiv. org/content/early/2015/11/13/031583

5. Caravagna G, Graudenzi A, Ramazzotti D, Sanz-Pamplona R, Sano LD, Mauri G, Moreno V, Antoniotti M, Mishra B (2016) Algorithmic methods to infer the evolutionary trajectories in cancer progression. Proc Natl Acad Sci USA 113(28):E4025-E4034. https://doi.org/10.1073/pnas.1520213113. http://www.pnas.org/content/113/28/ E4025

6. Constantinescu S, Szczurek E, Mohammadi P, Rahnenführer J, Beerenwinkel N (2015) TiMEx: a waiting time model for mutually exclusive cancer alterations. Bioinformatics. https://doi.org/10.1093/bioinformatics/ btv400

7. Crona K, Greene D, Barlow M (2013) The peaks and geometry of fitness landscapes. J Theor Biol 317:1-10. https://doi.org/10. 1016/j.jtbi.2012.09.028. http://www. sciencedirect.com/science/article/pii/ S0022519312005061

8. Datta RS, Gutteridge A, Swanton C, Maley CC, Graham TA (2013) Modelling the 
evolution of genetic instability during tumour progression. Evol Appl 6(1):20-33. https:// doi.org/10.1111/eva.12024. http://www. pubmedcentral.nih.gov/articlerender.fcgi? artid $=3567468 \&$ tool $=$ pmcentrez\& rendertype $=$ abstract

9. Desper R, Jiang F, Kallioniemi OP, Moch H, Papadimitriou CH, Schäffer AA (1999) Inferring tree models for oncogenesis from comparative genome hybridization data. J Comput Biol 6(1):37-51. http://view.ncbi.nlm.nih. gov/pubmed/10223663

10. Diaz-Uriarte $\mathrm{R}$ (2015) Identifying restrictions in the order of accumulation of mutations during tumor progression: effects of passengers, evolutionary models, and sampling. BMC Bioinf 16(41). https://doi.org/10.1186/ s12859-015-0466-7. http://www. biomedcentral.com/1471-2105/16/41/ abstract

11. Diaz-Uriarte R (2017) OncoSimulR: genetic simulation with arbitrary epistasis and mutator genes in asexual populations. Bioinformatics 33(12):1898-1899. https://doi.org/l0. 1093/bioinformatics/btx077. https://aca demic.oup.com/bioinformatics/article/33/

12/1898/2982052/OncoSimulR-geneticsimulation-with-arbitrary

12. Diaz-Uriarte R (2018) Cancer progression models and fitness landscapes: a many-tomany relationship. Bioinformatics 34 (5):836-844. https://doi.org/10.1093/bioin formatics/btx663. https://academic.oup. com/bioinformatics/article/34/5/836/ 4557185

13. Diaz-Uriarte R, Vasallo C (2019) Every which way? On predicting tumor evolution using cancer progression models. PLOS Comput Biol 15 (8):el007246. https://doi.org/10.1371/jour nal.pcbi.1007246. https://journals.plos.org/ ploscompbiol $/$ article?id=10.1371 $/$ journal. pcbi. 1007246

14. Ferretti L, Schmiegelt B, Weinreich D, Yamauchi A, Kobayashi Y, Tajima F, Achaz G (2016) Measuring epistasis in fitness landscapes: the correlation of fitness effects of mutations. J Theor Biol 396:132-143. https://doi.org/10.1016/j.jtbi.2016.01.037. http://www.sciencedirect.com/science/arti cle/pii/S0022519316000771

15. Gerrish PJ, Colato A, Perelson AS, Sniegowski PD (2007) Complete genetic linkage can subvert natural selection. Proc Natl Acad Sci USA 104(15):6266-6271. https://doi.org/10. 1073 pnas.0607280104

16. Gerstung M, Baudis M, Moch H, Beerenwinkel N (2009) Quantifying cancer progression with conjunctive Bayesian networks. Bioinformatics 25(21):2809-2815. https://doi.org/ 10.1093/bioinformatics/btp505. http:// www.bsse.ethz.ch/cbg/software/ct-cbn

17. Gerstung M, Eriksson N, Lin J, Vogelstein B, Beerenwinkel N (2011) The temporal order of genetic and pathway alterations in tumorigenesis. PLoS ONE 6(11):e27136. https://doi. org/10.1371/journal.pone.0027136. http:// www.bsse.ethz.ch/cbg/software/ct-cbn

18. Gillespie JH (1993) Substitution processes in molecular evolution. I. Uniform and clustered substitutions in a haploid model. Genetics 134 (3):971-981

19. Hosseini S-R, Diaz-Uriarte R, Markowetz F, Beerenwinkel N (2019) Estimating the predictability of cancer evolution. Bioinformatics 35(14):i389-i397. https://doi.org/10.1093/ bioinformatics/btz332. https://academic. oup.com/bioinformatics/article/35/14/ i389/5529151

20. Kauffman SA (1993) The origins of order: selforganization and selection in evolution, lst edn. Oxford University Press, New York. ISBN 978-0-19-507951-7

21. Kauffman SA, Weinberger ED (1989) The NK model of rugged fitness landscapes and its application to maturation of the immune response. J Theor Biol 141(2):211-245. https://doi.org/10.1016/S0022-5193(89)

80019-0. http://www.sciencedirect.com/sci ence/article/pii/S0022519389800190

22. Kingman JFC (1978) A simple model for the balance between selection and mutation. J Appl Probab 15(1):1-12. https://doi.org/10. 2307/3213231. https://www.cambridge. org/core/journals/journal-of-applied-proba bility/article/simple-model-for-the-balancebetween-selection-and-mutation/ 26726A951C67C23ADC2240066887ClF1

23. Mather WH, Hasty J, Tsimring LS (2012) Fast stochastic algorithm for simulating evolutionary population dynamics. Bioinformatics (Oxford, England) 28(9):1230-1238. https://doi.org/10.1093/bioinformatics/ bts130. http://www.ncbi.nlm.nih.gov/ pubmed/22437850

24. McFarland C (2014) The role of deleterious passengers in cancer. Ph.D. thesis, Harvard University. http://nrs.harvard.edu/urn-3: HUL.InstRepos:13070047

25. McFarland C, Mirny L, Korolev KS (2014) A tug-of-war between driver and passenger mutations in cancer and other adaptive processes. Proc Natl Acad Sci USA 111 (42):15138-15143. https://doi.org/10. 1101/003053. http://arxiv.org/pdf/ 1402.6354v1 \$backslash\$nhttp://arxiv.org/ abs/1402.6354

26. McFarland CD, Korolev KS, Kryukov GV, Sunyaev SR, Mirny LA (2013) Impact of 
deleterious passenger mutations on cancer progression. Proc Natl Acad Sci USA 110 (8):2910-2915. https://doi.org/10.1073/ pnas.1213968110. http://www.ncbi.nlm.nih. gov/pubmed/23388632

27. Montazeri H, Kuipers J, Kouyos R, Böni J, Yerly S, Klimkait T, Aubert V, Günthard HF, Beerenwinkel N, Study TSHC (2016) Largescale inference of conjunctive Bayesian networks. Bioinformatics 32(17):i727-i735. https://doi.org/10.1093/bioinformatics/ btw459. http://bioinformatics.oxfordjournals. org/content/32/17/i727

28. Neidhart J, Szendro IG, Krug J (2014) Adaptation in tunably rugged fitness landscapes: the rough Mount Fuji Model. Genetics 198 (2):699-721. https://doi.org/10.1534/ genetics.114.167668. http://www.genetics. org/content/198/2/699

29. Ochs IE, Desai MM (2015) The competition between simple and complex evolutionary trajectories in asexual populations. BMC Evol Biol 15(1):1-9. https://doi.org/10.1186/ s12862-015-0334-0. http://www. biomedcentral.com/1471-2148/15/55

30. Olde Loohuis L, Caravagna G, Graudenzi A, Ramazzotti D, Mauri G, Antoniotti M, Mishra B (2014) Inferring tree causal models of cancer progression with probability raising. PLOS ONE 9(10):el08358. https://doi.org/10. 1371/journal.pone.0108358. https:// journals.plos.org/plosone/article?id=10. 1371/journal.pone.0108358

31. Ramazzotti D, Caravagna G, Olde Loohuis L, Graudenzi A, Korsunsky I, Mauri G, Antoniotti M, Mishra B (2015) CAPRI: efficient inference of cancer progression models from cross-sectional data. Bioinformatics 31 (18):3016-3026. https://doi.org/10.1093/ bioinformatics/btv296. https://academic. oup.com/bioinformatics/article-lookup/doi/ 10.1093 /bioinformatics/btv296

32. Raphael BJ, Vandin F (2015) Simultaneous inference of cancer pathways and tumor progression from cross-sectional mutation data. J Comput Biol 22(00):250-264. https://doi. org/10.1089/cmb.2014.0161

33. Schoen DJ, Schultz ST (2019) Somatic mutation and evolution in plants. Annu Rev Ecol Evol Syst 50(1):49-73. https://doi.org/10. 1146/annurev-ecolsys-110218-024955. https://www.annualreviews.org/doi/10. 1146/annurev-ecolsys-110218-024955

34. Szabo A, Boucher KM (2008) Oncogenetic trees. In: Tan W-Y, Hanin L (eds) Handbook of cancer models with applications. World Scientific, pp 1-24. http://www.worldscibooks. $\mathrm{com} /$ lifesci/6677.html

35. Szendro IG, Schenk MF, Franke J, Krug J, de Visser JAGM (2013) Quantitative analyses of empirical fitness landscapes. J Stat Mech 2013 (01):P01005. https://doi.org/10.1088/ 1742-5468/2013/01/P01005. http:// stacks.iop.org/1742-5468/2013/i=01/ $\mathrm{a}=\mathrm{P} 01005$

36. Tomlinson IP, Novelli MR, Bodmer WF (1996) The mutation rate and cancer. Proc Natl Acad Sci USA 93(25):14800-14803

37. Zanini F, Neher RA (2012) FFPopSim: an efficient forward simulation package for the evolution of large populations. Bioinformatics 28 (24):3332-3333. https://doi.org/10.1093/ bioinformatics/bts633. http://webdav. tuebingen.mpg.de/ffpopsim/. http://bioin formatics.oxfordjournals.org/content/28/ 24/3332http://github.com/neherlab/ ffpopsim/

Open Access This chapter is licensed under the terms of the Creative Commons Attribution 4.0 International License (http://creativecommons.org/licenses/by/4.0/), which permits use, sharing, adaptation, distribution and reproduction in any medium or format, as long as you give appropriate credit to the original author(s) and the source, provide a link to the Creative Commons license and indicate if changes were made.

The images or other third party material in this chapter are included in the chapter's Creative Commons license, unless indicated otherwise in a credit line to the material. If material is not included in the chapter's Creative Commons license and your intended use is not permitted by statutory regulation or exceeds the permitted use, you will need to obtain permission directly from the copyright holder.

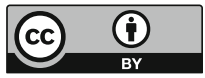

